\title{
A Nonparametric Bayesian Approach to Copula Estimation
}

\author{
Shaoyang Ning * \\ Department of Statistics, Harvard University, Cambridge, 02138, MA \\ shaoyangning@fas.harvard.edu \\ Neil Shephard ${ }^{\dagger}$ \\ Department of Economics and Department of Statistics, Harvard University, Cambridge, \\ 02138, MA \\ shephard@fas.harvard.edu
}

\begin{abstract}
We propose a novel Dirichlet-based Pólya tree (D-P tree) prior on the copula and based on the $\mathrm{D}-\mathrm{P}$ tree prior, a nonparametric Bayesian inference procedure. Through theoretical analysis and simulations, we are able to show that the flexibility of the D-P tree prior ensures its consistency in copula estimation, thus able to detect more subtle and complex copula structures than earlier nonparametric Bayesian models, such as a Gaussian copula mixture. Further, the continuity of the imposed D-P tree prior leads to a more favorable smoothing effect in copula estimation over classic frequentist methods, especially with small sets of observations. We also apply our method to the copula prediction between the S\&P 500 index and the IBM stock prices during the 2007-08 financial crisis, finding that D-P tree-based methods enjoy strong robustness and flexibility over classic methods under such irregular market behaviors.

KEY WORDS: copula, Pólya tree, nonparametric Bayes, Gaussian copula mixture model, kernel method
\end{abstract}

\section{INTRODUCTION}

The copula, as the "link" of a multivariate distribution to its marginals, has attracted growing interest in statistical research since Sklar (1959). By Sklar's Theorem, a copula characterizes the dependence structure between the marginal components. Therefore, the copula plays a central role in multivariate studies and has gained increasing popularity in application to fields such as risk analysis, insurance modeling, and hydrologic engineering (Nelsen 2007; Wu et al. 2014).

The estimation of copulas has been well studied in parametric and semi-parametric settings, but little work has been released on the nonparametric Bayesian inference. In this article, we propose

\footnotetext{
* Shaoyang Ning is a PhD student at Department of Statistics, Harvard University, Cambridge, MA 02138; email: shaoyangning@fas.harvard.edu.

${ }^{\dagger}$ Neil Shephard is a Professor of Economics and of Statistics at Department of Statistics and Department of Economics, Harvard University, Cambridge, MA 02138; email: shephard@fas.harvard.edu.
} 
a novel multi-partition Dirichlet-based Pólya tree (D-P tree) prior on the copula. Our D-P tree prior relaxes the binary partition constraints on earlier Pólya-tree-like priors but still preserves the favorable properties of the Pólya tree, including conjugacy and absolute continuity. Based on such a D-P tree prior, we provide a nonparametric Bayesian approach for copula estimation. Its consistency is validated through theoretical analysis.

The D-P tree prior overcomes the severe bias problem of previously proposed Pólya-tree-like priors, and the inconsistency issue of family-based nonparametric Bayesian approaches such as the Gaussian copula mixture (Dortet-Bernadet 2005) under model misspecification. Further, compared with classic nonparametric frequentist methods, including the empirical copula estimation and the kernel method, the D-P tree shows a more favorable smoothing effect, especially based on small sets of observations. We illustrate our new method by focusing on copula structure prediction between the S\&P 500 daily index and the IBM daily stock prices during the 2007-08 financial crisis. We find that D-P tree-based methods are rather robust and adaptive to irregular market behavior, especially in comparison with commonly-adopted parametric models and the empirical method.

Earlier parametric or semi-parametric methods often model copula functions within certain parametric copula families and estimate the parameters by maximum likelihood (ML). For marginals, either parametric or nonparametric estimations are usually adopted (Joe 1997, Jaworski et al. 2010 , Chen and Huang 2007; Oakes 1982 1986, Genest et al. 1995). However, these parametric or semiparametric methods suffer from the risk of severe bias when the model is misspecified, thus lack the flexibility to provide accurate estimation for more complex and subtle copula structures. In addition, copula itself is strictly-increasing-transform invariant (Schweizer and Wolff 1981). Thereby, under no further parametric assumptions, the rank statistics of data would preserve sufficient information required for the estimation. In light of these features, nonparametric methods seem to be more natural and coherent for the estimation of copula.

Most of the recent studies on nonparametric copula estimation focus on empirical methods (Jaworski et al. 2010; Deheuvels 1979), or kernel-related methods (Scaillet et al. 2007; Behnen et al. 1985; Gijbels and Mielniczuk 1990; Schuster 1985; Hominal and Deheuvels 1979; Devroye and Györfi 1985, Gasser and Müller 1979, John 1984; Müller 1991; Chen and Huang 2007). Current nonparametric Bayesian methods focus mainly on an infinite mixture of elliptical copula families such as the Gaussian or the skew-normal (Wu et al. 2014). Yet such models still have limitations: a heavy computational burden as they are implemented through MCMC, and an inconsistency when the model is misspecified, taking the infinite Gaussian copula mixture for a non-symmetric target 
copula as an instance. These motivate us to explore priors with conjugacy and more generality.

Note that here we focus mainly on the bivariate copula case to illustrate our method, and we will discuss higher-dimensional cases towards the end. Also, to concentrate on the estimation of copula structures itself, we assume that the marginals are known or can be accurately estimated. So equivalently, in our simulations, we are concerned mainly with marginally uniform data generated from copula distributions. Such an assumption is reasonable in that: (1) usually we have more information (either parametric or nonparametric) on the marginals of the data for the estimation; (2) multivariate data are exponentially enriched when considered marginally, providing higher resolution for accurate estimation. Yet we will discuss the scenarios where marginal distributions are to be empirically estimated.

The article is organized as follows: in Section 2, we establish some notation and review previous attempts for copula estimation based on the Pólya tree prior and their limitations. In Section 3. we introduce the proposed D-P tree prior and the procedure for copula inference. In Section 4 , we elaborate on properties of the D-P tree. Section 5 provides a simulation-based evaluation of our method in comparison with other common copula estimation methods. In Section 6, we provide an application of our method to the analysis of a bivariate stock-index copula structure. We discuss the copula estimation with unknown marginal distributions and the higher-dimensional cases in Section 7, Section 8 concludes the article.

\section{THE QUASI-PÓLYA TREE PRIOR ON COPULA}

\subsection{The Pólya Tree Prior}

Our focus here is on finding Pólya-tree-like priors placed on a copula. The Pólya tree (PT) prior is a tractable case of a tail-free process (Ferguson 1974), which also includes the Dirichlet process (DP) as a special case. But unlike the Dirichlet process, the Pólya tree delivers absolutely continuous measures with probability one by certain choices of the hyper-parameters, which is the attraction for our applications.

Following the definition by Lavine (1992) (Appendix A.2), suppose we have a probability measure $\mathcal{P}$ that follows a Pólya tree prior, i.e., $\mathcal{P} \sim P T(\Pi, \mathcal{A})$. The conjugacy of the Pólya tree follows in that, with one observation $Y \mid \mathcal{P} \sim \mathcal{P}$, the posterior $\mathcal{P} \mid Y$ still follows a Pólya tree distribution denoted by $P T(\Pi, \mathcal{A} \mid Y)$ with the hyper-parameters updated by

$$
\alpha_{\epsilon} \mid Y= \begin{cases}\alpha_{\epsilon}+1 & \text { if } Y \in B_{\epsilon} \\ \alpha_{\epsilon} & \text { otherwise. }\end{cases}
$$


In practice, to ensure the absolute continuity of measures given by the Pólya tree prior, the hyper-parameters usually take as $\alpha_{\epsilon}=z m^{2}$ at $m$-th level of the partition, where $z$ is a fixed constant, and the infinite-level Pólya tree is approximated by terminating the sampling process from $\operatorname{PT}(\Pi, \mathcal{A})$ at finite level $M$.

Therefore, the PT can be intuitively viewed as a smoothed random histogram, and enjoys favorable features such as conjugacy and absolute continuity. Note that Hanson (2006) studied the finite mixture of Pólya trees; Paddock et al. (2003) and Wong et al. (2010) extended the classic PT with randomized partitions to embraces higher flexibility; Filippi and Holmes (in press) applied Pólya tree to independence test based on the Bayes factor. So it seems promising to start with the $\mathrm{PT}$ in search of a more favorable nonparametric prior for Bayesian copula inference.

\subsection{Dortet-Bernadet's quasi-Pólya tree prior on copula}

To our knowledge, Dortet-Bernadet (2005) made the first attempt to apply the PT prior to the inference of a bivariate copula on $I=[0,1] \times[0,1]$. At each level, each square partition $B_{\epsilon}$ is split into four sub-partitions $\left\{B_{\epsilon 0}, B_{\epsilon 1}, B_{\epsilon 2}, B_{\epsilon 3}\right\}$ by dyadic partitions on its margins. Thereby, a partition of $I$ is obtained by $\Pi=\left\{B_{\epsilon}\right\}, \epsilon \in\{\emptyset, 0,1,2,3,00,01,02,03 \ldots\}$, demonstrated by the left panel of Figure 1 .
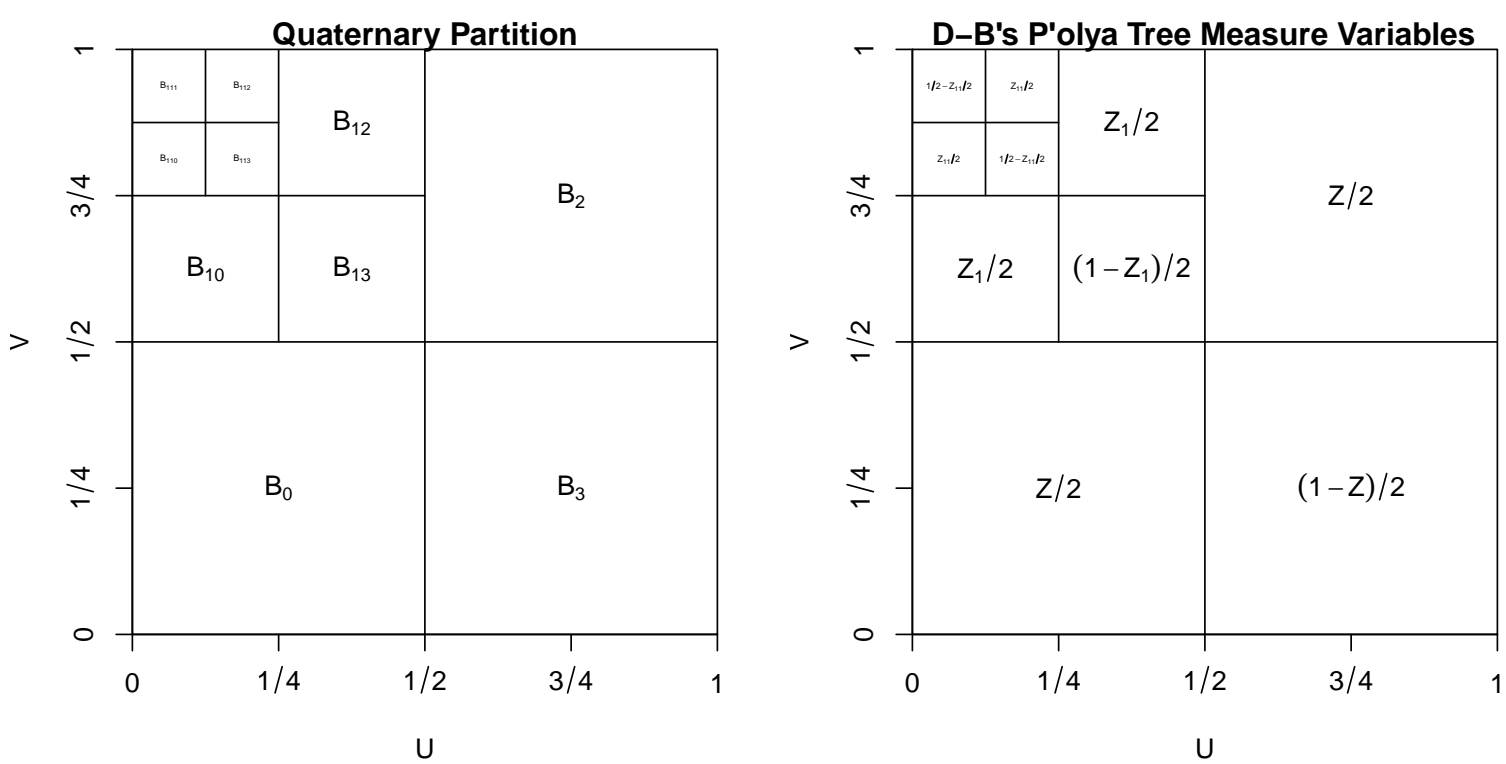

Figure 1: The quaternary partition (left) on the support $[0,1]^{2}$ of a bivariate copula and the parametrization of Dortet-Bernadet's quasi-Pólya tree prior (right).

The Pólya-tree-like probability measure $\mathcal{P}$, which we call a quasi-Pólya tree prior, is defined by 
independent variables $\mathcal{Z}=\left\{Z_{\epsilon}\right\}$, hyper-parameters $\mathcal{A}=\left\{\alpha_{\epsilon 0}, \alpha_{\epsilon 1}\right\}$, where $Z_{\epsilon} \sim \operatorname{Beta}\left(\alpha_{\epsilon 0}, \alpha_{\epsilon 1}\right)$ and

$$
\mathcal{P}\left(B_{\epsilon=\epsilon_{1} \epsilon_{2} \ldots \epsilon_{m}}\right)=\left(\prod_{j=1 ; \epsilon_{j}=0 \text { or } \epsilon_{j}=2}^{m} Z_{\epsilon_{1} \epsilon_{2} \ldots \epsilon_{j-1}} / 2\right)\left\{\prod_{j=1 ; \epsilon_{j}=1 \text { or } \epsilon_{j}=3}^{m}\left(1-Z_{\epsilon_{1} \epsilon_{2} \ldots \epsilon_{j-1}}\right) / 2\right\} .
$$

The posterior-like hyper parameters are updated as:

$$
\alpha_{\epsilon 0} \mid Y=\left\{\begin{array}{ll}
\alpha_{\epsilon 0}+1 & \text { if } Y \in B_{\epsilon 0} \cup B_{\epsilon 2}, \\
\alpha_{\epsilon 0} & \text { otherwise; }
\end{array} \quad \alpha_{\epsilon 1} \mid Y= \begin{cases}\alpha_{\epsilon 1}+1 & \text { if } Y \in B_{\epsilon 1} \cup B_{\epsilon 3}, \\
\alpha_{\epsilon 1} & \text { otherwise. }\end{cases}\right.
$$

Unfortunately, Dortet-Bernadet's quasi-Pólya tree prior performs rather unsatisfactorily even in simple bivariate Gaussian copula case. As shown in Figure 2, where we estimate the Gaussian copula with $\rho=0.9$ based on $N=10,000$ data points and approximation level $M=10$, the "grid" effect is severe for such a quasi-Pólya Tree prior, leading to considerable bias for estimation.
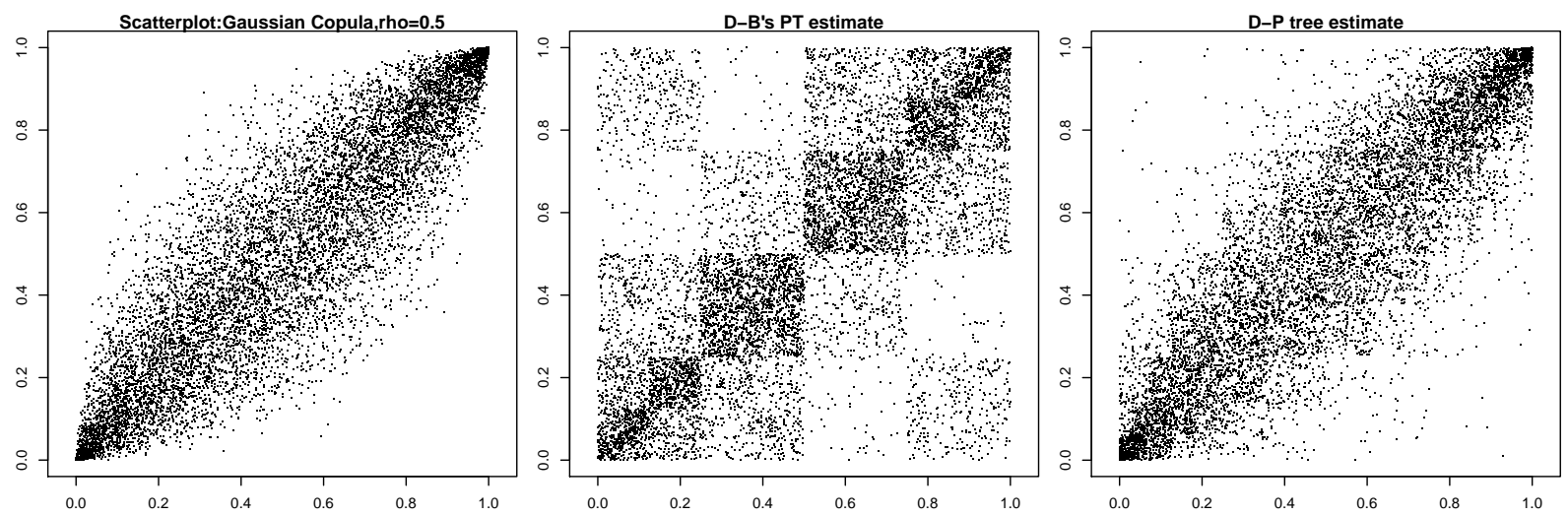

Figure 2: Scatterplots comparing the Gaussian copula estimates: the Gaussian copula (left), the quasi-Pólya tree (middle) and the D-P tree (right) priors.

In fact, Dortet-Bernadet's Pólya tree prior deviates from the classic Pólya tree in that it mixes a binary partition with a quaternary partition across levels. It does not preserve the features of PT such as the conjugacy, so the posterior-like update is rather ad hoc. Further, it puts strong constraints on its dependence structure by combining the two diagonal dyadic sub-partitions at each level when updating the hyper-parameters for posterior, which causes severe bias when the true copula is heavily asymmetric in the super-partition at the previous level.

\section{OUR APPROACH: DIRICHELET-BASED PÓLYA TREE}

\subsection{The Dirichlet-based Pólya Tree (D-P tree)}

One natural way to remedy the inflexibility in the design of the quasi-Pólya tree is to adopt the more flexible Dirichlet distribution for measure variables $\left(Z_{\epsilon}\right)$ in place of the much-constrained Beta distribution in the classic PT. Here we first give the Dirichlet-based Pólya tree a general definition: 
Definition 1. Let $\Omega$ be a separable measurable space. We say a partition $\Pi=\left\{B_{\epsilon}\right\}$ of $\Omega$ is one of its measurable tree partitions if

- the subpartitions at level $m+1\left\{B_{\epsilon_{1} \ldots \epsilon_{m+1}}\right\}$ is refinement of previous level $\left\{B_{\epsilon_{1} \ldots \epsilon_{m}}\right\}$;

- $\Pi=\left\{B_{\epsilon}\right\}$ generates measurable sets of $\Omega$.

Definition 2. Let $\Omega$ be a separable measurable space and $\Pi=\left\{B_{\epsilon}\right\}$ be one of its measurable tree partitions. A random probability measure $\mathcal{P}$ is said to have a Dirichlet-based Pólya tree distribution, or $D$-P tree prior, with parameters $(\Pi, \mathcal{A})$, written $\mathcal{P} \sim \operatorname{DPT}(\Pi, \mathcal{A})$, if there exists non-negative numbers $\mathcal{A}=\left\{\alpha_{\epsilon}\right\}$ and random variables $\mathcal{Z}=\left\{\boldsymbol{Z}_{\epsilon}\right\}$ such that the following hold:

- all the random vectors in $\mathcal{Z}$ are independent;

- for every $m=1,2, \ldots$ and every sequence $\epsilon=\epsilon_{1} \epsilon_{2} \ldots \epsilon_{m}, Z_{\epsilon}=\left(Z_{\epsilon 0}, \ldots, Z_{\epsilon k_{\epsilon}}\right) \sim \operatorname{Dirichlet}\left(\alpha_{\epsilon 0}, \ldots, \alpha_{\epsilon k_{\epsilon}}\right)$, with $B_{\epsilon}=\cup_{i=0}^{k_{\epsilon}} B_{\epsilon i}$ and $k_{\epsilon}$ the number of subpartitions in $B_{\epsilon}$;

- for every $\epsilon, \mathcal{P}\left(B_{\epsilon=\epsilon_{1} \epsilon_{2} \ldots \epsilon_{m}}\right)=\left(\prod_{j=1}^{m} Z_{\epsilon_{1} \epsilon_{2} \ldots \epsilon_{j}}\right)$.

The D-P tree prior still falls into the general class of tail-free process, as the random variables for measures are independent across different partition levels. Yet rather than constraining on binary partitions and beta distributions, the D-P tree adopts a more flexible partition structure and, accordingly, the Dirichlet-distributed variables for the measures, which preserves similar properties to the classic Pólya tree prior.

\subsection{Conjugacy and Posterior Updating}

Adapting the D-P tree prior to bivariate copula estimation, we constrain the $\mathrm{D}-\mathrm{P}$ tree on $\Omega=I=$ $[0,1] \times[0,1]$, with the quaternary dyadic partition $\Pi=\left\{B_{\epsilon 0}, B_{\epsilon 1}, B_{\epsilon 2}, B_{\epsilon 3}\right\}$, which repeats Section

2.2 . but now the hyper-parameters $\mathcal{A}=\left\{\alpha_{\epsilon 0}, \alpha_{\epsilon 1}, \alpha_{\epsilon 2}, \alpha_{\epsilon 3}\right\}$ and random variables $\left(Z_{\epsilon 0}, Z_{\epsilon 1}, Z_{\epsilon 2}, Z_{\epsilon 3}\right) \sim$ $\operatorname{Dirichlet}\left(\alpha_{\epsilon 0}, \alpha_{\epsilon 1}, \alpha_{\epsilon 2}, \alpha_{\epsilon 3}\right)$, as illustrated in Figure 3. From now on, without further specification, we focus only on the D-P tree prior with such a quaternary dyadic partition parametrization, though all results can be generalized.

Such D-P tree prior preserves the conjugacy property of original Pólya tree, thus with $\mathcal{P} \sim$ $\operatorname{DPT}(\Pi, \mathcal{A})$ and an observation $Y \mid \mathcal{P} \sim \mathcal{P}$, the posterior $\mathcal{P} \mid Y$ can be readily updated.

Proposition 1 (Conjugacy). Let $\mathcal{P}$ be a measure on $I=[0,1] \times[0,1]$, and an observation $Y \mid \mathcal{P} \sim \mathcal{P}$. Suppose $\mathcal{P}$ follows a $D$-P tree prior, as $\mathcal{P} \sim D P T(\Pi, \mathcal{A})$, with the quaternary partition $\Pi=\left\{B_{\epsilon}\right\}$ 

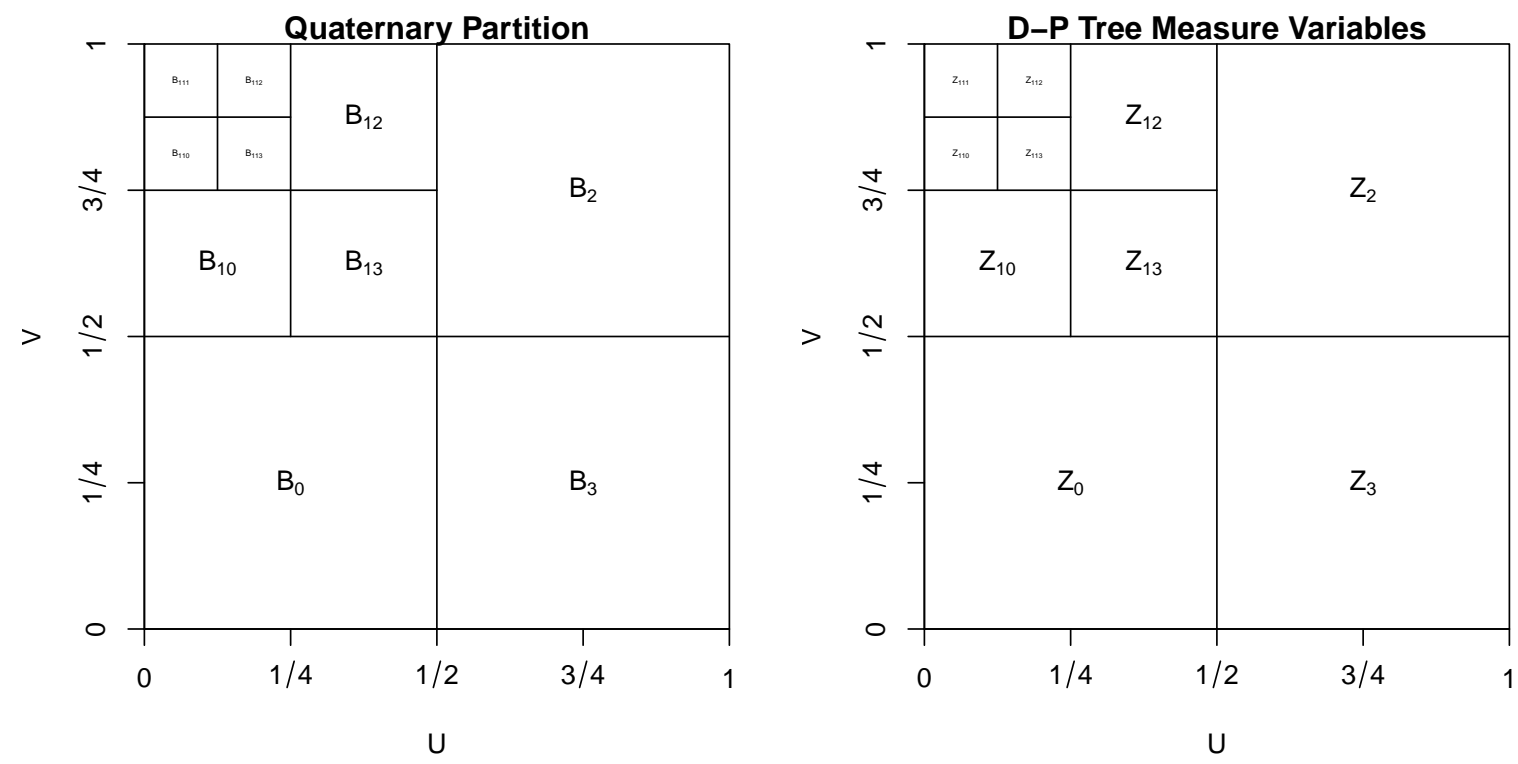

Figure 3: The quaternary partition (left) on the support $[0,1]^{2}$ of a bivariate copula and the parametrization of Dirichelet-based tree (D-P tree) prior (right).

and Dirichlet-distributed random variables $\mathcal{Z}=\left\{Z_{\epsilon}\right\}$ and hyper-parameters $\mathcal{A}=\left\{\alpha_{\epsilon 0}, \alpha_{\epsilon 1}, \alpha_{\epsilon 2}, \alpha_{\epsilon 3}\right\}$. Then the posterior $\mathcal{P} \mid Y \sim D P T(\Pi, \mathcal{A} \mid Y)$, where, for $i=0,1,2,3$,

$$
\alpha_{\epsilon i} \mid Y= \begin{cases}\alpha_{\epsilon i}+1 & \text { if } Y \in B_{\epsilon i} \\ \alpha_{\epsilon i} & \text { otherwise }\end{cases}
$$

Proof: $p(\mathcal{Z} \mid Y) \propto p(Y \mid \mathcal{Z}) p(\mathcal{Z}) \propto \prod_{j=1}^{\infty} Z_{\epsilon_{1} \ldots \epsilon_{j}} \prod Z_{\epsilon}^{\alpha_{\epsilon}} \propto \prod Z_{\epsilon}^{\alpha_{\epsilon}+I_{Y \in B_{\epsilon}}} \square$.

For $N$ i.i.d. observations $\boldsymbol{Y}=\left(Y_{1}, Y_{2}, \ldots, Y_{N}\right)$, the posterior update for multiple observations is rather intuitive and straightforward: at each level of the partitions, the hyper-parameter $\alpha_{\epsilon}$ associated with the specific partition $B_{\epsilon}$ is incremented by the number of observations falling in that partition, denoted by $n_{\epsilon}$, where $n_{\epsilon}=\sum_{i=1}^{N} I_{Y_{i} \in B_{\epsilon}}$. Simply put: $\alpha_{\epsilon} \mid \boldsymbol{Y}=\alpha_{\epsilon}+n_{\epsilon}$.

\subsection{Copula Estimation by the D-P Tree Prior}

For the copula estimation, suppose we have $N$ i.i.d. observations $\boldsymbol{Y}=\left(Y_{1}, Y_{2}, \ldots, Y_{N}\right)$ from an unknown copula distribution $C$, i.e., $Y_{1}, Y_{2}, \ldots, Y_{N} \stackrel{\text { i.i.d. }}{\sim} C$. We assume that $C$ follows a D-P tree prior, i.e., $C \sim \operatorname{DPT}(\Pi, \mathcal{A})$, where we take $\Pi$ to be the quaternary partition on the unit square $[0,1] \times[0,1]$ and $\mathcal{A}=\left\{\alpha_{\epsilon}: \alpha_{\epsilon_{1} \ldots \epsilon_{m}}=m^{2}\right\}$. By Proposition 1, the posterior $C \mid \boldsymbol{Y} \sim D P T(\Pi, \mathcal{A} \mid \boldsymbol{Y})$, where $\mathcal{A} \mid \boldsymbol{Y}=\left\{\alpha: \alpha_{\epsilon_{1} \ldots \epsilon_{m}}=m^{2}+n_{\epsilon}\right\}$.

Therefore, the D-P tree posterior on copula strongly resembles the construction of a histogram of the observations, but regularized by the imposed prior. Later we will show the choice of hyper- 
parameters, as in $\mathcal{P} \sim \operatorname{DPT}\left(\Pi, \mathcal{A}=\left\{\alpha_{\epsilon}: \alpha_{\epsilon_{1} \ldots \epsilon_{m}}=m^{2}\right\}\right)$, ensures generating absolutely continuous measures centered on the uniform distribution, and thus the posterior then can be viewed as a shrunk version of the histogram.

In practice, we approximate the infinite-level D-P tree prior with its $M$-level approximation $\mathcal{P}$ :

Definition 3. For a probability measure $\mathcal{P}$ such that $\mathcal{P} \sim D P T(\Pi, \mathcal{A})$, with the same notation as in Definition 2. its $M$-level approximation $\mathcal{P}_{M}$ is, for any measurable set $B \in\left\{B_{\epsilon=\epsilon_{1} \epsilon_{2} \ldots \epsilon_{M}}\right\}$,

$$
\mathcal{P}_{M}(B)=\left(\prod_{j=1}^{M} Z_{\epsilon_{1} \epsilon_{2} \ldots \epsilon_{j}}\right) \frac{\mu(B)}{\mu\left(B_{\epsilon=\epsilon_{1} \epsilon_{2} \ldots \epsilon_{M}}\right)},
$$

where $\mu$ is the uniform measure on $\Pi$.

\section{PROPERTIES OF D-P TREE}

\subsection{Equivalence to the Pólya Tree}

We first show that, through a re-parametrization, the D-P tree prior on the unit square with the quaternary partition complies with a classic Pólya tree by sequentially combining the quaternary partitions to binary partitions.

Proposition 2 (Equivalence to the Pólya tree). Given a $D$-P tree prior on $I=[0,1] \times[0,1]$ with the quaternary partition $\Pi=\left\{B_{\epsilon}\right\}$ and Dirichlet-distributed random variables $\mathcal{Z}=\left\{Z_{\epsilon}\right\}$ and hyperparameters $\mathcal{A}=\left\{\alpha_{\epsilon 0}, \alpha_{\epsilon 1}, \alpha_{\epsilon 2}, \alpha_{\epsilon 3}\right\}$, an equivalent Pólya tree prior with binary partition $\tilde{\Pi}=\left\{\tilde{B}_{\eta}\right\}$ and Beta-distributed $\tilde{\mathcal{Z}}=\left\{\tilde{Z}_{\eta}\right\}$ and hyper-parameters $\tilde{\mathcal{A}}=\left\{\tilde{\alpha}_{\eta 0}, \tilde{\alpha}_{\eta 1}\right\}$ can be constructed as

$$
\begin{gathered}
\tilde{B}_{\eta_{1} \eta_{2} \ldots \eta_{2 k}}=B_{\epsilon_{1} \epsilon_{2} \ldots \epsilon_{k}}, \quad \tilde{B}_{\eta_{1} \eta_{2} \ldots \eta_{2 k+1}}=B_{\epsilon_{1} \epsilon_{2} \ldots \epsilon_{k}\left(2 \eta_{2 k+1}\right)} \cup B_{\epsilon_{1} \epsilon_{2} \ldots \epsilon_{k}\left(2 \eta_{2 k+1}+1\right)}, \\
\tilde{\alpha}_{\eta_{1} \eta_{2} \ldots \eta_{2 k}}=\alpha_{\epsilon_{1} \epsilon_{2} \ldots \epsilon_{k}}, \quad \tilde{\alpha}_{\eta_{1} \eta_{2} \ldots \eta_{2 k+1}}=\alpha_{\epsilon_{1} \epsilon_{2} \ldots \epsilon_{k}\left(2 \eta_{2 k+1}\right)}+\alpha_{\epsilon_{1} \epsilon_{2} \ldots \epsilon_{k}\left(2 \eta_{2 k+1}+1\right)}
\end{gathered}
$$

where $k=0,1, \ldots, \epsilon_{i}=2 \eta_{2 i-1}+\eta_{2 i}, i=1,2, \ldots k$.

This result follows directly from the property of representing a Dirichlet distribution by independent Gamma distributions, and the independence property between the represented Beta and Gamma distributions. With such equivalence, some of the favorable features of the classic Pólya tree prior can be naturally extended to the D-P tree.

\subsection{Continuity of D-P Tree Prior}

Here we show that the D-P tree prior inherits the feature of generating absolute continuous probability measures under certain constraints on the hyper-parameters $\mathcal{A}$. 
Proposition 3 (Absolute continuity). A $D$-P tree prior on $I=[0,1] \times[0,1]$ with the quaternary partition $\Pi=\left\{B_{\epsilon}\right\}$ and Dirichlet-distributed random variables $\mathcal{Z}=\left\{Z_{\epsilon}\right\}$ and hyper-parameters $\mathcal{A}=\left\{\alpha_{\epsilon 0}, \alpha_{\epsilon 1}, \alpha_{\epsilon 2}, \alpha_{\epsilon 3}\right\}$ generates an absolute continuous probability measure on $I$ with probability one when hyper-parameters on the m-level $\alpha_{\epsilon_{1} \ldots \epsilon_{m}} \propto O\left(m^{1+\delta}\right), \delta>0$.

Further, with $\boldsymbol{Y}=\left(Y_{1}, Y_{2}, \ldots, Y_{N}\right) \mid P \stackrel{\text { i.i.d. }}{\sim} \mathcal{P}, \mathcal{P} \sim D P T(\Pi, \mathcal{A})$, the posterior $\operatorname{DPT}(\Pi, \mathcal{A} \mid \boldsymbol{Y})$ also generates an absolute continuous probability measure with probability one.

The results follow from Theorem 1.121 and Lemma 1.124 in (Schervish 1995). Thereby, as we implied earlier in Section 3.3, the canonical hyper-parameter choice, i.e., $\alpha_{\epsilon_{1} \ldots \epsilon_{m}}=m^{2}$ will lead to a D-P tree prior that yields absolutely continuous random probability measures, which justifies the smoothing effect of the D-P tree prior in copula estimation.

\subsection{Consistency of the D-P Tree Posterior}

Suppose we have $N$ i.i.d. observations $\boldsymbol{Y}=\left\{Y_{1}, \ldots, Y_{N}\right\}$ generated from true copula distribution $C$. For copula estimation, we assume $Y_{i} \mid C \stackrel{i . i . d .}{\sim} \mathcal{C}$, with a D-P tree prior $\left.C \sim D P T(\Pi, \mathcal{A}\}\right)$. Let $\mathcal{P}_{M}$ be the M-level approximation of $C$ and $\mathcal{A}$ be canonical, i.e., the m-level hyper-parameter $\alpha_{\epsilon_{1} \ldots \epsilon_{m}}=m^{2}$.

For the approximated posterior $\mathcal{P}_{M} \mid \boldsymbol{Y}$, we have the point-wise convergence to the target copula distribution in terms of any measurable set in the unit square:

Proposition 4 (Point-wise convergence). For any measurable set $B \subset I=[0,1] \times[0,1]$, with $N \propto O\left(M^{3+\eta}\right), \eta>0$, then $E\left(\left(\mathcal{P}_{M}(B) \mid \boldsymbol{Y}\right)-C(B)\right) \rightarrow 0, \operatorname{var}\left(\mathcal{P}_{M}(B) \mid \boldsymbol{Y}\right)=O\left(\frac{M}{N}\right)$, therefore $\mathcal{P}_{M}(B) \mid \boldsymbol{Y} \stackrel{p}{\rightarrow} C(B)$.

Notice that here we require that the sample size goes to infinity with a higher order than $O\left(M^{3}\right)$, which leaves the variance of our D-P posterior $\left(O\left(\frac{M}{N}\right)\right)$ in a higher order than the empirical copula estimator $\left(O\left(\frac{1}{N}\right)\right)$. In fact, by introducing such a D-P tree prior, we sacrifice some asymptotic statistical efficiency in exchange of the continuity of our estimator. Also, as shown in the simulation results later, we gain some advantages in prediction precision with small sets of observations.

If we put smoothness constraints on the target distribution, we can have similar convergence results uniformly on $I$ for the posterior, and further the consistency of the posterior.

Proposition 5 (Consistency). If $C \in C^{1}([0,1] \times[0,1])$, for $B \subset$ I measurable, $\sup _{B} \mid E\left(\mathcal{P}(\mathcal{B})_{M} \mid \boldsymbol{Y}\right)-$ $C \mid=\max \left\{O\left(\frac{M}{\sqrt{N} \gamma(M)}\right), O\left(\frac{M^{3}}{N \gamma(M)}\right)\right\} ; \sup _{B} \operatorname{var}\left(\mathcal{P}(\mathcal{B})_{M} \mid \boldsymbol{Y}\right)=O\left(\frac{M}{N \gamma(M)}\right)$, where $\gamma(M) \sim \min _{C\left(B_{M}\right)>0} C\left(B_{M}\right)$.

Further, with $N \propto O\left(2^{10 M} M^{2+\eta}\right), \eta>0, \forall \delta>0$ as $M \rightarrow \infty, P\left(d_{T V}\left(\mathcal{P}_{M}, C\right) \geq \delta \mid Y\right) \rightarrow 0$. Note that $d_{T V}$ is the total variation distance between probability measures. 
Specifically, we refine the order of convergence for several classic copula distributions, which, in practice, may serve as general guidance for the choice of partition level $M$ based on sample size $N$.

Proposition 6. The order requirement for the uniform convergence of specific target copulas:

1. For a lower-bounded copula density, i.e., $c \geq \xi>0, \gamma(M) \geq 2^{-2 M} \xi$, thus $N \propto O\left(M^{2+\eta} 2^{4 M}\right)$;

2. For a bivariate Gaussian copula, $\gamma(M) \geq \Phi^{2}\left(\sqrt{1-|\rho|} \Phi^{-1}\left(2^{-M}\right)\right) \sqrt{\frac{1-|\rho|}{1+|\rho|}}$, thus $N \propto O\left(M^{2+\eta} 2^{4 M}\right)$.

Proofs of these results are provided in Appendix B. Such convergence properties ensure the consistency of the estimation based on the D-P tree prior, giving the $\mathrm{D}-\mathrm{P}$ tree prior advantages over family-based estimation methods under model misspecification.

\section{SIMULATION EXPERIMENTS}

\subsection{Evaluation: Common Copulas}

To evaluate the performance of our copula estimation procedure, we conduct simulation studies based on common copulas as listed in Supplementary Material S.1 with various parameter settings, among which Gaussian, Student's and Gumbel are symmetric while the skew-normal is asymmetric.

For each simulation, the procedure is as follows: we first draw i.i.d. data samples from true copula $C$ with the size of $N$, denoted by $\boldsymbol{Y}$; then we follow the procedure described in Section 3.3 for the posterior inference on $C$; once posterior $\operatorname{DPT}(\Pi, \mathcal{A} \mid \boldsymbol{Y})$ is obtained, we draw 10,000 posterior predictive samples from $\mathcal{P}_{M} \mid \boldsymbol{Y}$ to plot the scatterplots, shown in Figure 44, 6. Note that without further clarification, all simulations are done with approximation level $M=10$.

\subsubsection{Posterior Scatterplots}

We first report the scatterplots of the posterior predictive draws compared with i.i.d. draws from the target copula distributions based on sample size $N=10,000$, as shown by Figure 5. The plots come in pairs with the left one showing i.i.d. draws from the true copula and the right one i.i.d. predictive draws from the posterior D-P tree to compare. In most cases, our proposed D-P tree prior works well. The difference between our predictive density and the true copula is mild, with exceptions in highly correlated Gumbel case $(a=4)$ and highly truncated case (skew-normal, $\rho=0.9, \boldsymbol{\alpha}=(-10,50))$.

In light of this, we increase the original sample size to $N=100,000$ (Figure 6). The overdispersion in low-density area and the grid effect in highly correlated area are eliminated, which corresponds with our asymptotic properties described in Section 4.3 . 

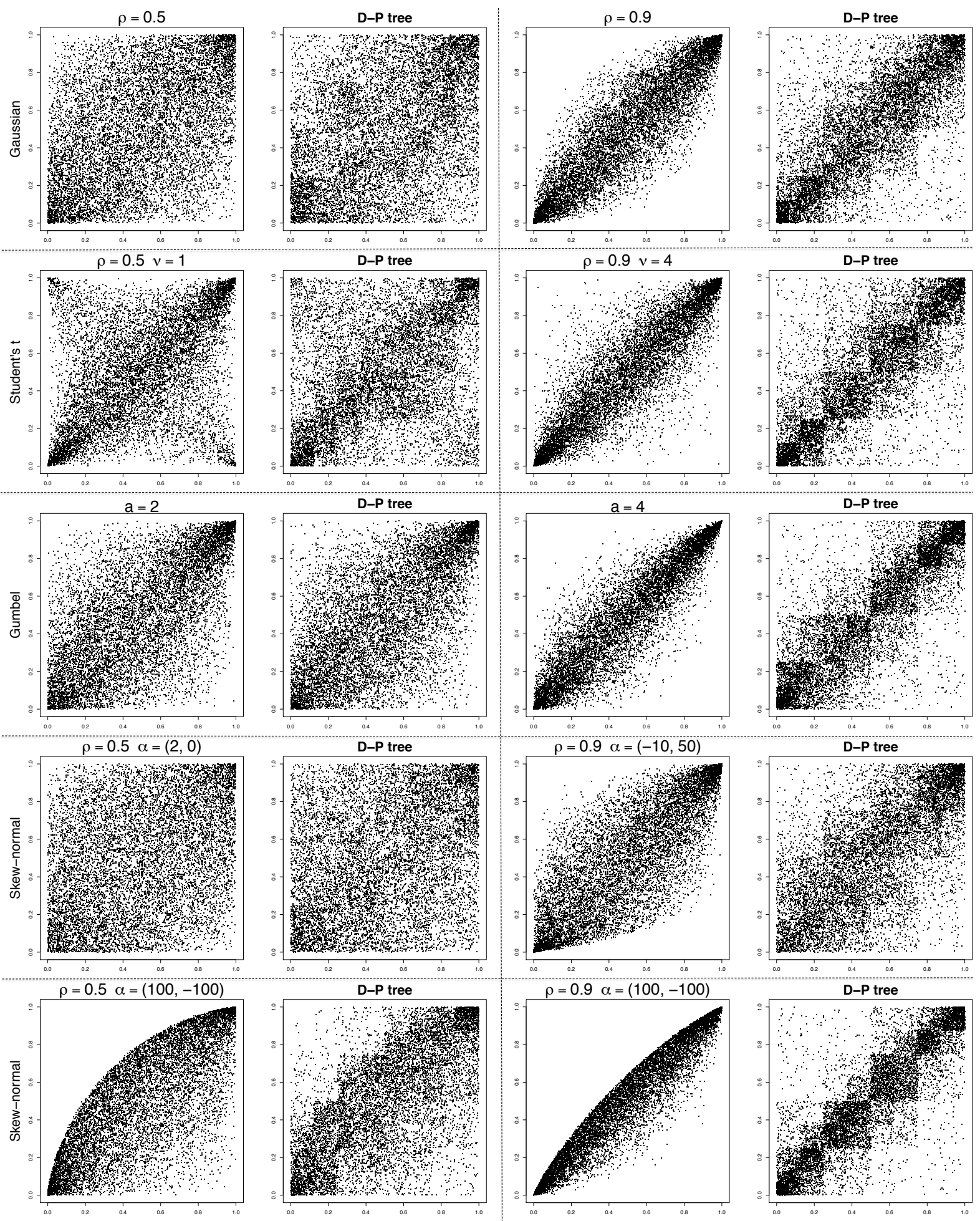

Figure 4: Scatterplots of i.i.d. draws from the true copula distribution (left) vs. the D-P tree posterior (right): sample size $N=1,000$, partition level $M=10$. 

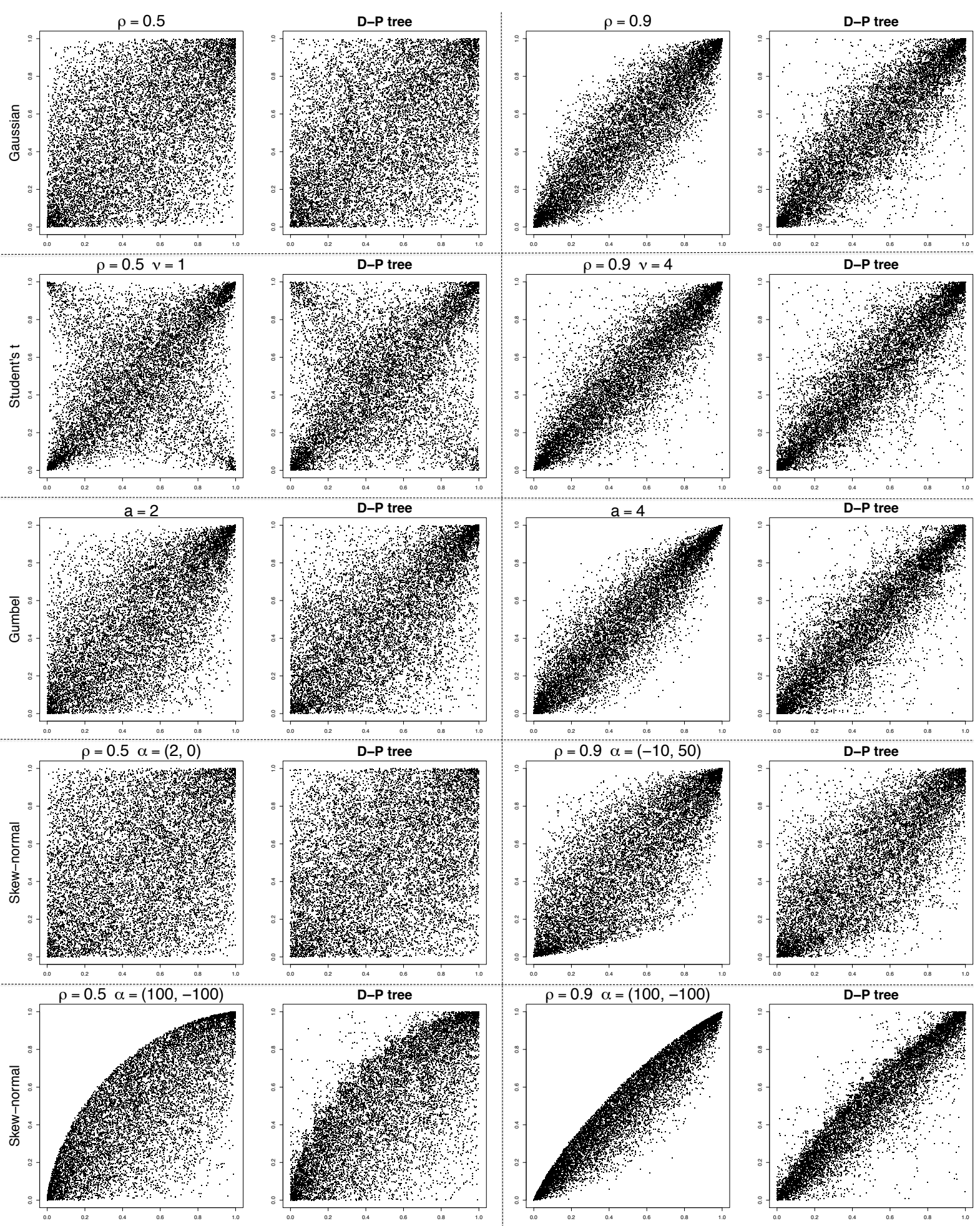

Figure 5: Scatterplots of i.i.d. draws from the true copula distribution (left) vs. the D-P tree posterior (right): sample size $N=10,000$, partition level $M=10$. 

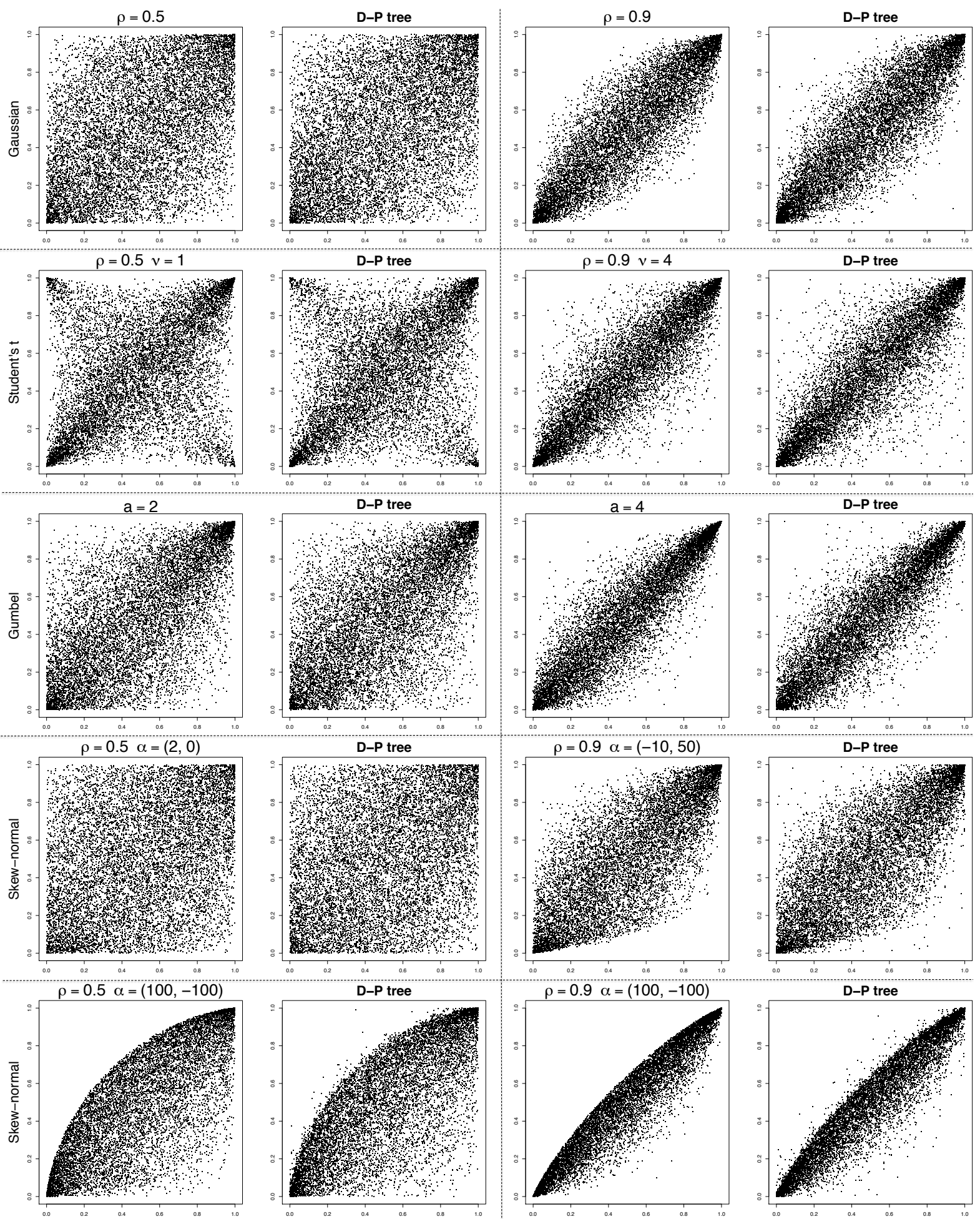

Figure 6: Scatterplots of i.i.d. draws from the true copula distribution (left) vs. the D-P tree posterior (right): sample size $N=100,000$, partition level $M=10$. 
We also explored cases with the more challenging $N=1,000$. Here the asymptotic conditions break, with $m=O(1)$ and $N / M^{3}=1$. Thereby, for more complex copula structures, the D-P tree prior is prone to loss of sensitivity due to the reduced sample size, which results in the rather unfavorable grid effect as in Figure 4.

\subsubsection{Kullback-Leibler Divergence}

We further evaluate our method for copula density estimation quantitatively using the KullbackLeibler (K-L) divergence of our estimates from the true copula:

$$
D_{K L}\left(C \| \mathcal{P}_{M}\right)=\mathrm{E}\left\{\log \left(c / p_{M}\right)\right\}=\iint \log \frac{c(u, v)}{p_{M}(u, v)} d C(u, v),
$$

where $p_{M}$ is the density for $\mathcal{P}_{M}, c$ is the true copula density, and the expectation is taken over the true copula distribution $C$.

We vary the sample size $N$ from 0 to 100,000. For each sample size, we draw 1,000 posterior densities from the D-P tree posterior and calculate the K-L divergence using Monte-Carlo method. The mean and variance of the K-L divergence are reported in Table 1 for various copula families, as well as the box plots across various sample sizes by Figure 7 .

Among all copula families, both mean and variance of the K-L divergence of the D-P tree posterior converge to zero, showing the evidence of consistency in posterior. Specifically the K-L divergence would drop below 0.10 when the sample size is increased to 10,000 , and the variance goes to 0 , consistent with the convergence claims in section 4.3 .

To explore the convergence rate, we fit both $D_{K L} \sim \log N$ and $\log D_{K L} \sim \log N$ with linear regression. The fitted curves are shown in Figure 7 by red and green respectively. The green curve gives almost perfect fitting, indicating the convergence rate is in the order of $N^{\alpha}, \alpha<0$, though theoretical verification is still required.

\subsection{Comparison with Existing Methods}

Here we compare our method with several existing nonparametric methods for copula estimation.

\subsubsection{Comparison with Nonparametric Bayesian Methods}

We first compare our method with the infinite Gaussian mixture copula model (Wu et al. 2014). For copula distribution $C$, we have the prior $C \sim \sum_{i=1}^{\infty} w_{i} C_{g}\left(\rho_{i}\right)$, where $C_{g}$ indicates the bivariate Gaussian copula, and the weight $w_{i} \stackrel{i . i . d .}{\sim} U[0,1]$ and the correlation $\rho_{i} \stackrel{\text { i.i.d. }}{\sim} U[-1,1]$. Such a model is the most common one among existing nonparametric Bayesian methods which focus on mixture models based on a specific copula family. 


\begin{tabular}{rr|llllll}
\hline \hline \multicolumn{1}{r}{$N$} & 0 & 10 & 100 & 1,000 & 10,000 & 100,000 \\
\hline \hline & $\rho$ & \multicolumn{5}{|c}{ Gaussian } \\
\hline & 0.50 & $0.82(0.19)$ & $0.54(0.05)$ & 0.25 & 0.13 & 0.07 & 0.04 \\
& 0.90 & $1.50(0.35)$ & $0.83(0.02)$ & 0.48 & 0.22 & 0.10 & 0.05 \\
\hline \hline$\rho$ & $\nu$ & \multicolumn{5}{c}{ Student's t } \\
\hline 0.50 & 1.00 & $1.01(0.18)$ & $0.70(0.03)$ & 0.38 & 0.21 & 0.10 & 0.05 \\
0.90 & 1.00 & $0.86(0.21)$ & $0.60(0.05)$ & 0.24 & 0.14 & 0.07 & 0.04 \\
0.50 & 4.00 & $1.72(0.33)$ & $1.04(0.03)$ & 0.61 & 0.30 & 0.13 & 0.06 \\
0.90 & 4.00 & $1.53(0.37)$ & $1.01(0.05)$ & 0.48 & 0.23 & 0.10 & 0.05 \\
\hline \hline & $a$ & & \multicolumn{5}{c}{ Gumbel } \\
\hline & 2.00 & $1.01(0.21)$ & $0.83(0.07)$ & 0.30 & 0.16 & 0.08 & 0.04 \\
& 4.00 & $1.66(0.39)$ & $1.04(0.05)$ & 0.53 & 0.25 & 0.11 & 0.05 \\
\hline \hline$\rho$ & $\alpha$ & & \multicolumn{5}{c}{ skew-normal } \\
\hline 0.50 & $(2,0)$ & $0.72(0.15)$ & $0.44(0.03)$ & 0.23 & 0.12 & 0.07 & 0.04 \\
0.50 & $(-10,50)$ & $0.91(0.19)$ & $0.52(0.02)$ & 0.31 & 0.18 & 0.09 & 0.05 \\
0.90 & $(-10,50)$ & $1.22(0.27)$ & $0.65(0.01)$ & 0.40 & 0.21 & 0.10 & 0.05 \\
0.90 & $(50,0)$ & $1.09(0.22)$ & $0.65(0.04)$ & 0.37 & 0.16 & 0.08 & 0.04 \\
0.50 & $(100,-100)$ & $1.35(0.30)$ & $0.82(0.02)$ & 0.48 & 0.26 & 0.14 & 0.07 \\
0.90 & $(100,-100)$ & $2.13(0.39)$ & $1.39(0.04)$ & 0.86 & 0.46 & 0.20 & 0.08 \\
\hline \hline
\end{tabular}

Table 1: Estimated K-L divergence of the D-P tree posterior from various targets, with standard errors (SE). Note that we leave out SEs (all 0.00) for $N \geq 100$.

Here, we focus on the non-symmetric skew-normal copulas as the data generating copulas. The simulations are carried out with the sample size varying from $N=1,000$ to $N=100,000$, and the K-L divergences of the estimates from the true target copula distribution for both methods are calculated with Monte-Carlo method. We report in Table 2 the cases where the skew-normal copula is highly non-symmetric, and thus the Gaussian mixture model is severely misspecified. More comprehensive simulation results on various target copulas are shown in Supplementary Material S.2. For less non-symmetric copulas $(\alpha=(2,0),(-10,50),(50,0))$, the Gaussian mixture model dominates due to its parametric nature, yet for these highly non-symmetric cases $(\alpha=(100,-100))$ in Table 2, the D-P tree shows a gradually increasing advantage as the data size increases. The results also illustrate the inconsistency issue of the Gaussian mixture model, as its K-L divergence from the data-generating model remains stable $(0.17,0.16)$ as sample size increases, while the converging trend for the D-P tree posterior is evident.

\subsubsection{Comparison with Nonparametric Frequentist Methods}

We select three classic nonparametric methods in frequentist settings in comparison with our D-P tree. Suppose $Y_{i}=\left(U_{i}, V_{i}\right) \stackrel{\text { i.i.d. }}{\sim} C$ : 

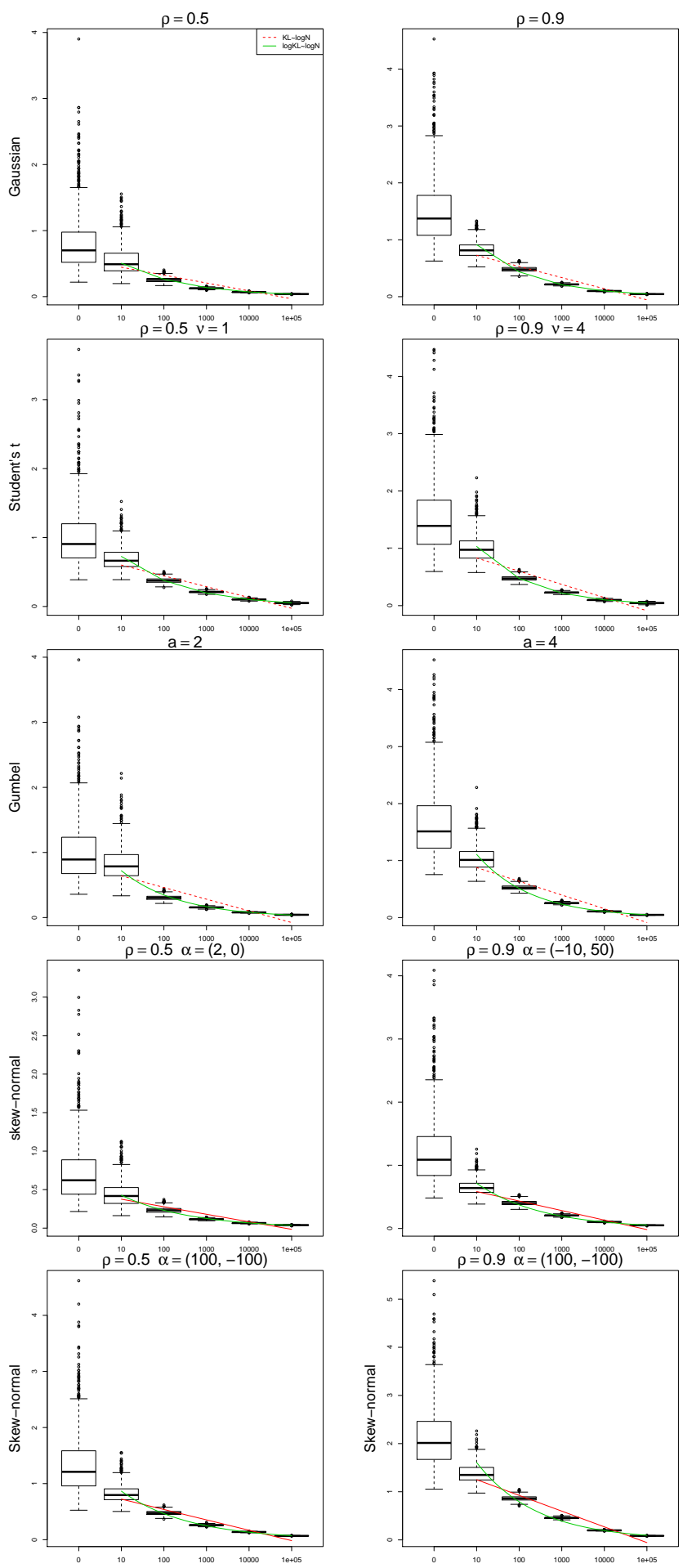

Figure 7: Box-plots of the K-L divergence of the D-P tree posterior from the target copulas against the sample size $N$ : the solid green line showing a linear fit of $\log (\mathrm{KL}) \sim \log (N)$.

- The empirical estimator: $\hat{C}_{\text {emp }}(u, v)=\frac{1}{N} \sum_{i=1}^{N} I_{U_{i} \leq u} I_{V_{i} \leq v}$.

- The histogram estimator: $\hat{C}_{\text {hist }}\left(B_{\epsilon}\right)=\frac{n_{\epsilon}}{N}$, where $B_{\epsilon}$ is the partition at the highest level. 


\begin{tabular}{rc|cc|cc|cc}
\hline \hline \multirow{2}{*}{$\rho$} & \multicolumn{6}{|c}{$N$} \\
\cline { 3 - 8 } & & \multicolumn{2}{|c}{1,000} & \multicolumn{2}{c}{10,000} & 100,000 \\
\cline { 3 - 8 } & & D-P & GM & D-P & GM & D-P & GM \\
\hline 0.50 & $(100,-100)$ & 0.26 & 0.17 & 0.14 & 0.17 & 0.07 & 0.17 \\
0.90 & $(100,-100)$ & 0.46 & 0.16 & 0.20 & 0.16 & 0.08 & 0.16 \\
\hline \hline
\end{tabular}

Table 2: Comparison of the K-L divergence between the D-P tree (D-P) and the Gaussian mixture (GM) model for highly non-symmetric skew-normal target copulas.

- The independent Gaussian kernel estimator (Jaworski et al. 2010):

$$
\hat{C}_{k e r}(u, v)=\frac{1}{N} \sum_{i=1}^{N} \Phi\left\{\frac{\Phi^{-1}(u)-\Phi^{-1}\left(U_{i}\right)}{h}\right\} \Phi\left\{\frac{\Phi^{-1}(v)-\Phi^{-1}\left(V_{i}\right)}{h}\right\},
$$

where we make the choice of $h=N^{-\frac{1}{5}}$, following Silverman's rule of thumb for the choice of window width.

- The D-P tree posterior mean estimator: for a fair comparison, we use the mean distribution from the D-P tree posterior as the Bayesian estimator by the D-P tree, i.e., $\hat{C}_{D-P}=\mathrm{E}(C \mid \boldsymbol{Y})$.

We define several measurements for the distance between the estimator and the target distribution. For density estimation, besides the K-L divergence, we also include the commonly adopted MISE (Mean Integrated Squared Error) based on the averaged $L_{2}$-norm between the estimated density function and the truth:

$$
\operatorname{MISE}(\hat{c})=\mathrm{E}\left[\iint_{[0,1] \times[0,1]}\{c(u, v)-\hat{c}(u, v)\}^{2} d u d v\right] .
$$

Here $c$ is the target copula density and $\hat{c}$ is its estimator.

For the distance measurement of the distribution, we extend the MISE for density to the $M I S E_{C}$ (Mean Integrated Squared Error for Cumulative functions):

$$
M I S E_{C}(\hat{C})=\mathrm{E}\left[\iint_{[0,1] \times[0,1]}\{C(u, v)-\hat{C}(u, v)\}^{2} d u d v\right],
$$

where $\hat{C}$ is the estimated copula function, $C$ is the true copula. We also have a distance measure specifically targeting the grid-based estimation methods, the $M S E_{g}$ :

$$
M S E_{g}(\hat{C})=\mathrm{E}\left[\frac{1}{2^{2 M}} \sum_{i, j=1}^{2^{M}}\left\{C\left(B_{i j}\right)-\hat{C}\left(B_{i j}\right)\right\}^{2}\right]
$$

where $\left\{B_{i j}\right\}$ are partitions on $[0,1] \times[0,1]$, and $M$ is the maximum partition level. Note that all the expectations in the measures defined above are taken over all possible data samples 
The simulations are carried out with the sample size varying from $N=10$ to $N=10,000$ for a good look at the convergence trend. We again focus mainly on heavily non-symmetric skew-normal copulas $(\alpha=(-50,10),(100,-100))$. For each parameter setting, we first draw $N$ i.i.d. samples from the true copula distribution, obtain the copula estimates by three frequentist methods and the D-P tree posterior mean estimator; then we repeat this process 50 times to obtain the Monte-Carlo approximation of the measures as defined above. Note that for the empirical copula estimation, the estimated distribution is discrete, thus the density distance measures not applicable; for the histogram estimator, due to the discrepancy in the supports between the target and the estimated distributions, the K-L divergence is not applicable. To ensure computational efficiency, we report the results based on the approximation level $M=8$, and to maintain comparability, we take the same maximum partition level for the histogram estimation method. Comprehensive numeric results are shown in Table $\mathrm{S} .2, \mathrm{~S} .5$ in Supplementary Material S.2. Here we report mainly the results under the parameter setting $\rho=0.5, \alpha=(100,-100)$ in Table 3 as exemplary for our conclusions.

\begin{tabular}{r|cccc|cccc}
\hline \hline \multirow{2}{*}{$N$} & \multicolumn{5}{|c|}{ K-L } & \multicolumn{4}{c}{$\sqrt{M I S E}$} \\
\cline { 2 - 8 } & D-P Tree & Empirical & Kernel & Hist. & D-P Tree & Empirical & Kernel & Hist. \\
\hline 10 & 0.528 & NA & 0.528 & Inf & 1.365 & NA & 2.190 & 71.788 \\
20 & 0.473 & NA & 0.428 & Inf & 1.163 & NA & 2.657 & 56.726 \\
50 & 0.386 & NA & 0.314 & Inf & 1.050 & NA & 1.177 & 36.757 \\
100 & 0.349 & NA & 0.261 & Inf & 1.159 & NA & 1.347 & 25.723 \\
500 & 0.222 & NA & 0.166 & Inf & 1.072 & NA & 1.398 & 11.665 \\
1,000 & 0.184 & NA & 0.136 & Inf & 0.894 & NA & 0.703 & 8.078 \\
5,000 & 0.112 & NA & 0.090 & Inf & 0.703 & NA & 0.516 & 3.601 \\
10,000 & 0.089 & NA & 0.076 & Inf & 0.701 & NA & 0.769 & 2.600 \\
\hline \hline & \multicolumn{9}{|c|}{} & $\sqrt{M I S E_{C}}$ & & $\sqrt{M S E_{g}}$ & \\
\hline 10 & 0.072 & 0.118 & 0.091 & 0.117 & 0.054 & 0.321 & 0.054 & 0.321 \\
20 & 0.065 & 0.082 & 0.068 & 0.082 & 0.054 & 0.230 & 0.055 & 0.230 \\
50 & 0.044 & 0.057 & 0.050 & 0.057 & 0.054 & 0.151 & 0.054 & 0.151 \\
100 & 0.037 & 0.041 & 0.038 & 0.041 & 0.054 & 0.113 & 0.054 & 0.113 \\
500 & 0.018 & 0.017 & 0.017 & 0.017 & 0.053 & 0.070 & 0.053 & 0.070 \\
1,000 & 0.013 & 0.012 & 0.013 & 0.012 & 0.053 & 0.062 & 0.053 & 0.062 \\
5,000 & 0.007 & 0.006 & 0.007 & 0.006 & 0.053 & 0.055 & 0.053 & 0.055 \\
10,000 & 0.005 & 0.004 & 0.005 & 0.004 & 0.053 & 0.054 & 0.053 & 0.054 \\
\hline \hline
\end{tabular}

Table 3: Comparison of various distance measures between the D-P tree posterior mean estimator and frequentist estimators for the skew-normal copula with parameter $\rho=0.5, \alpha=(100,-100)$.

In general, the D-P tree posterior mean estimator performs competitively well compared with all three frequentist nonparametric methods and consistently across various measures. Notably, the D-P tree posterior estimation appears advantageous over other methods with small sets of observations, showcasing a preferably strong smoothing effect induced by the D-P tree prior. 
Both the D-P tree and the kernel estimation show drastic advantages in copula density estimation over empirical and histogram methods, as the empirical copula fails to yield density estimator and the histogram estimator gives severely poor density approximation due to the discrepancy in the support. Though both methods take advantage of the smoothing effect in estimation density, under the MISE measurement, the D-P tree dominates kernel method across almost all sample sizes while giving close figures under the K-L divergence.

As for copula distribution estimation, the D-P tree shows a strong advantage over other methods in both measures under scenarios of smaller sample size, which indicates the more favorable continuity feature of the D-P tree prior. When the sample size increases, the neutralizing effect of the D-P tree prior slows down the convergence of the posterior, and thereby, the empirical and histogram estimators catch up in figures. Yet still, up to $N=10,000$, the $\mathrm{D}-\mathrm{P}$ tree gives close distances as the empirical and the histogram methods, and consistently dominates the kernel method.

\section{REAL DATA APPLICATION}

For real data analysis, we apply our method to the S\&P 500 daily index and the IBM daily stock prices over the past 20 years (Jan 1, 1994 to Dec 31, 2014, available from https://finance. yahoo.com) and aim to estimate their dependence structure with the copula model. We adopt both the cross-validation and the rolling prediction schemes to evaluate the performance of our method, as described below in detail. We assess three methods under both prediction schemes for comparison: (1) the D-P tree posterior mean with canonical prior; (2) the Gaussian copula; (3) the Student's t copula. For the rolling prediction assessment, we also include (4) the independent Gaussian kernel estimator; (5) the empirical copula estimator, and (6) the D-P tree posterior mean with historic-data-induced prior. Typically, investment groups have focused on using methods (2), (3) and particularly (5) in practice for risk management.

\subsection{Cross-validation}

We first conduct cross-validation to evaluate the prediction ability of our method. Let the joint daily prices for two stocks be $\left\{\left(y_{i}^{1}, y_{i}^{2}\right), i=1, \ldots, T\right\}$, where $T=5,288$, and the returns of $\log$ price $\left\{r_{i}^{j}=\log y_{i}^{j}-\log y_{i-1}^{j}, i=2 \ldots T, j=1,2\right\}$. Marginally, we fit the commonly adopted GARCH $(1,1)$ model: $r_{i}^{j}=\sigma_{i}^{j} \epsilon_{i}^{j},\left(\sigma_{i}^{j}\right)^{2}=\alpha_{0}^{j}+\alpha_{1}^{j}\left(\sigma_{i-1}^{j}\right)^{2}+\beta_{1}^{j}\left(\epsilon_{i-1}^{j}\right)^{2}$, where the innovations $\left\{\epsilon_{i}^{j}\right\}$ are independent with $\mathrm{E}\left(\epsilon_{i}^{j}\right)=0$ and $\operatorname{var}\left(\epsilon_{i}^{j}\right)=1$. Further, we assume the distribution of the innovations is time- 
invariant and put the copula model on their joint distribution $F\left(\epsilon_{i}^{1}, \epsilon_{i}^{2}\right)=C\left(F^{1}\left(\epsilon_{i}^{1}\right), F^{2}\left(\epsilon_{j}^{2}\right)\right)$, where $\left(\epsilon_{i}^{1}, \epsilon_{i}^{2} \stackrel{i \text { i.i.d. }}{\sim} F\right.$, and $F^{1}$ and $F^{2}$ are the marginal distributions.

We apply the proposed D-P tree (canonical) prior to the copula estimation based on the fitted innovations from the GARCH model $\left\{\left(\hat{\epsilon}_{i}^{1}, \hat{\epsilon}_{i}^{2}\right)\right\}$. Here we use the empirical estimation for the marginals, as discussed in Section 7.2 . Figure 8 compares the scatterplots of the fitted errors (normalized by marginals) and the draws from the D-P tree posterior. We observe no apparent discrepancy between the data and the fitted model.
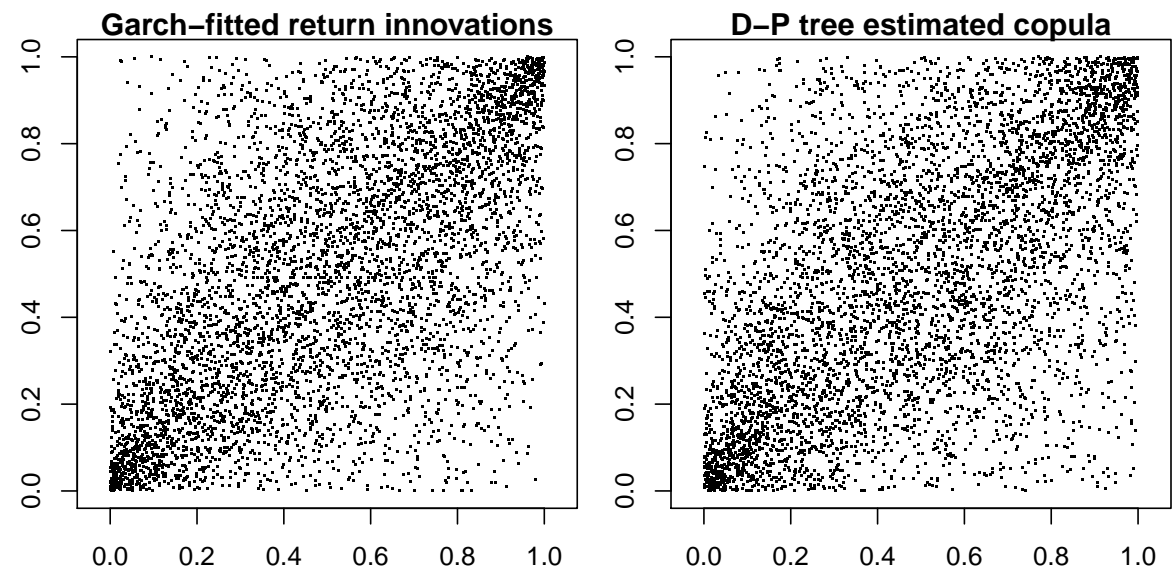

Figure 8: Scatterplots comparing the GARCH-fitted joint return innovations (left, normalized by the empirical marginal distributions) and random draws from the D-P tree estimated copula (right).

For comparison, we also fit the innovations with the bivariate Gaussian and Student's t copula models respectively, as described in Appendix S.1. The estimates are obtained by maximum likelihood estimation.

To assess the effectiveness of such estimation methods, we adopt the cross-validation scheme to get the estimation of the prediction errors. We randomly divide the marginally GARCH-fitted innovations into 10 sets and each time use one set as the testing set and the rest as the training set. For prediction errors, we adopt the Monte-Carlo estimation for cross entropy $-E_{c}(\log (\hat{c}))$, which is equivalent to the K-L divergence of the estimated copula density from the assumed truth up to a constant. Here, $c$ and $\hat{c}$ denote the true copula density and the estimated one respectively.

Table 4 gives the mean K-L divergence based on 10-fold cross-validation for the three methods in comparison. As it shows, the D-P tree prior outperforms the Gaussian copula narrowly, while the Student's t shows an advantage over the other two methods. The result is unsurprising as the distribution of stock returns are notable for the heavy-tail dependence features and the Student's t is thus expected to give good fittings. Nevertheless, when parametric models are misspecified under 
the Gaussian copula model, nonparametric methods such as the D-P tree prior is still advantageous.

\begin{tabular}{r|rrr}
\hline \hline & D-P tree & Gaussian $(\hat{\rho}=0.59)$ & Student's t $(\hat{\rho}=0.60, \hat{\nu}=6.5)$ \\
\hline Cross entropy & -0.210 & -0.209 & -0.224 \\
\hline \hline
\end{tabular}

Table 4: Comparison of the mean prediction errors based on the K-L divergence between the D-P tree prior, the Gaussian copula and the Student's t copula. Note that the more negative the numbers, the better the prediction performance.

\subsection{Rolling Prediction}

To mimic the practical prediction scenario, we also evaluate the prediction power of our method under the time-rolling prediction scheme, that is, we predict the future copula structure within a certain window of time based on the most recent observations.

Specifically, we set a training length of $T_{t r}$, a testing set length of $T_{t e}$, a rolling estimation window of length $t_{e}$, and a prediction window of length $t_{p}$. Firstly, we use the daily price time series of the two stocks $\left\{y_{t}^{1}: t=1, \ldots, T_{t r}\right\}$ and $\left\{y_{t}^{2}, t=1, \ldots, T_{t r}\right\}$ as the training set for the marginal GARCH-model fitting. Consistent with common practical prediction scenarios, we fix such fitted GARCH model and obtain the fitted innovations for the training set $\left\{\left(\hat{\epsilon}_{t}^{1}, \hat{\epsilon}_{t}^{2}\right), t=1, \ldots, T_{t r}\right\}$, and the predicted innovations for the test set $\left\{\left(\hat{\epsilon}_{t}^{1}, \hat{\epsilon}_{t}^{2}\right), t=T_{t r}+1, \ldots, T_{t r}+T_{t e}\right\}$. Then, we conduct the rolling prediction of the copula structure based on these estimates. For each rolling step, we apply the proposed D-P tree-based method with both the canonical non-informative prior and the historic-data-induced prior to the most recent $t_{e}$-fitted/predicted innovations and estimate the future copula structure of length $t_{p}$. Here we implicitly assume the i.i.d. property of the innovations within the estimation and prediction windows combined of length $\left(t_{e}+t_{p}\right)$. This is reasonable in that the copula structure is usually stable within a certain length of time. We repeat such rolling prediction $T_{t e} / t_{p}$ times until the whole testing length $\left(T_{t r}+1\right.$ to $\left.T_{t r}+T_{t e}\right)$ is covered.

Here we focus on the data of the period covering the 2007-08 financial crisis (i.e., the testing set covering July, 2007 to July, 2009) to highlight the flexibility and robustness of nonparametric methods over traditional parametric models. We set $T_{t r}=500, T_{t e}=500$, and vary $t_{e} \in\{10,20,50,100,250\}, t_{p} \in\{1,50\}$ and report both the average log-likelihood $\frac{1}{T_{t e}} \sum_{t=1}^{T_{t e}} \log \hat{c_{t}}$ (equivalent to negative KL divergence plus a constant), and the square root of average $M I S E_{C}=$ $\frac{1}{T_{t e}} \sum_{t=1}^{T_{t e}} \operatorname{MISE} E_{C}\left(\hat{C}_{t}\right)$ as the measures for prediction accuracy (Table 5 . Note that for historicdata-based D-P tree prior, we adopt the posterior of a canonical D-P tree prior updated by the data from testing set $\left(i=1, \ldots, T_{t r}-t_{e}\right)$ with each down-weighted by 0.1 . We also carry out the same prediction scheme with other four methods for comparison. 


\begin{tabular}{|c|c|c|c|c|c|c|c|c|c|c|c|c|c|}
\hline \multirow{2}{*}{$t_{e}$} & \multirow{2}{*}{$t_{p}$} & \multicolumn{6}{|c|}{ Average log-likelihood } & \multicolumn{6}{|c|}{$\sqrt{M I S E_{C}}$} \\
\hline & & D-PT & D-PTw & Emp. & Kernel & Gauss. & $\mathrm{t}$ & $\mathrm{D}-\mathrm{PT}$ & D-PTw & Emp. & Kernel & Gauss. & $\mathrm{t}$ \\
\hline 10 & 1 & -0.002 & 0.133 & NA & -0.052 & 0.094 & 0.086 & 0.312 & 0.300 & 0.338 & 0.305 & 0.300 & 0.301 \\
\hline 20 & 1 & 0.046 & 0.135 & NA & 0.030 & 0.141 & 0.139 & 0.310 & 0.301 & 0.328 & 0.305 & 0.299 & 0.300 \\
\hline 50 & 1 & 0.096 & 0.141 & NA & 0.044 & 0.141 & 0.143 & 0.310 & 0.304 & 0.322 & 0.306 & 0.299 & 0.299 \\
\hline 100 & 1 & 0.155 & 0.176 & NA & 0.096 & 0.154 & 0.160 & 0.309 & 0.306 & 0.318 & 0.306 & 0.298 & 0.299 \\
\hline 250 & 1 & 0.173 & 0.178 & NA & 0.105 & 0.153 & 0.158 & 0.306 & 0.306 & 0.312 & 0.304 & 0.298 & 0.298 \\
\hline 10 & 50 & 0.023 & 0.138 & NA & -0.075 & -0.340 & -0.128 & 0.082 & 0.062 & 0.123 & 0.099 & 0.078 & 0.074 \\
\hline 20 & 50 & 0.051 & 0.137 & NA & 0.003 & 0.009 & 0.028 & 0.082 & 0.064 & 0.102 & 0.091 & 0.071 & 0.071 \\
\hline 50 & 50 & 0.113 & 0.156 & NA & 0.058 & 0.106 & 0.113 & 0.066 & 0.060 & 0.070 & 0.068 & 0.066 & 0.066 \\
\hline 100 & 50 & 0.155 & 0.173 & NA & 0.067 & 0.137 & 0.139 & 0.060 & 0.058 & 0.063 & 0.062 & 0.063 & 0.063 \\
\hline 250 & 50 & 0.175 & 0.181 & NA & 0.108 & 0.150 & 0.158 & 0.057 & 0.057 & 0.058 & 0.058 & 0.061 & 0.061 \\
\hline
\end{tabular}

Table 5: Comparison of the prediction performance in the average log-likelihood (the higher the numbers, the better the prediction) and the $\operatorname{MISE}_{C}$ (the lower, the better) between various methods: the D-P tree posterior mean with the canonical prior (D-PT), the D-P tree with the historic-data-induced prior (D-PTw), the empirical copula (Emp.), the kernel estimator (Kernel), the Gaussian copula (Gauss.) and the Student's t copula (t) models.

Generally, both the D-P-tree-based methods show strong advantages over other methods by the log-likelihood loss in almost all settings, and by $\sqrt{M I S E_{C}}$ under a longer prediction window $t_{p}=50$ (where the distribution-based measure $\sqrt{M I S E_{C}}$ is more valid due to multiple testing samples) and a larger prediction set $t_{e} \geq 50$. Such results verify the robustness and adaptiveness of the D-P tree-based methods to irregular market behaviors when classic parametric models are terribly misspecified. Further, by incorporating the historic data into the prior, the D-PTw method enjoys a strong boost in prediction accuracy, and dominates other methods in most of the scenarios. Admittedly, more data are used by the D-PTw for inference than other methods in comparison. Nevertheless, it is exactly the showcase of the strength of Bayesian methods where historic or empirical information is readily concocted into priors to help.

\section{DISCUSSION}

\subsection{Copula Normalizing}

One problem with most nonparametric copula estimation methods including the D-P tree prior is that the posterior marginal does not always follow a uniform distribution. Suppose $\mathcal{P} \sim$ $\operatorname{DPT}(\Pi, \mathcal{A} \mid \boldsymbol{Y})$, then marginally $\mathcal{P}([0,1 / 2] \times[0,1]) \sim \operatorname{Beta}\left(\alpha_{0}+n_{0}+\alpha_{1}+n_{1}, \alpha_{2}+n_{2}+\alpha_{3}+n_{3}\right)$, which deviates from 0.5 by the randomness. Though, when the sample size $N$ is large, as shown by Proposition 4 the posterior density would have marginals close to uniforms, thus approximate a proper copula density, the issue of normalizing posterior density to proper copula density still needs addressing. Here we provide several methods to carry out the correction. 


\subsubsection{Ad Hoc Correction}

Suppose we have $\mathcal{P}^{*} \sim \operatorname{DPT}(\Pi, \mathcal{A} \mid Y)$ and $\mathcal{P}_{M}^{*}$ is its M-level approximation with a $2^{M} \times 2^{M}$ grid density. To normalize its marginals to the uniforms, we need to restrain

$$
\mathcal{P}_{M}^{*}\left(\left[k / 2^{M},(k+1) / 2^{M}\right] \times[0,1]\right)=\mathcal{P}_{M}^{*}\left([0,1] \times\left[k / 2^{M},(k+1) / 2^{M}\right]\right)=1 / 2^{M},
$$

$k=0,1, \ldots, 2^{M}-1$; i.e., the column sum and row sum of the $2^{M} \times 2^{M}$ grid density to be $1 / 2^{M}$. One way to realize this is to randomly select $2 \cdot 2^{M}-1$ grids and manipulate their values to fit (7).

As $\mathcal{P}_{M}^{*}$ is close to $C$ when the sample size is large, the marginals of $\mathcal{P}_{m}$ would not be too far away from a uniform. Thus the ad hoc correction would not cause severe deviation from the posterior density $\mathcal{P}^{*}$.

\subsubsection{Inverse Transform on the Marginals}

Another way of normalization is to apply the PIT (Probability Inverse Transform) to the marginals of $\mathcal{P}_{M}^{*}$. Factorize the M-level approximate posterior density by $\mathcal{P}_{M}^{*}([0, x] \times[0, y])=C_{\mathcal{P}_{M}^{*}}\left(F_{x, \mathcal{P}_{M}^{*}}(x), F_{y, \mathcal{P}_{M}^{*}}(y)\right)$, where $F_{x, \mathcal{P}_{M}^{*}}$ and $F_{y, \mathcal{P}_{M}^{*}}$ are the marginal CDFs of $\mathcal{P}_{M}^{*}$, and $C_{\mathcal{P}_{M}^{*}}$ is their copula. By transforming $(x, y) \rightarrow\left(F_{x, \mathcal{P}_{M}^{*}}(x), F_{y, \mathcal{P}_{M}^{*}}(y)\right)=(u, v)$, we have the normalized distribution $\tilde{\mathcal{P}}_{M}^{*}$ :

$$
\tilde{\mathcal{P}}_{M}^{*}([0, u] \times[0, v])=C_{\mathcal{P}_{M}^{*}}(u, v)=\mathcal{P}_{M}^{*}\left(\left[0, F_{x, \mathcal{P}_{M}^{*}}^{-1}(u)\right] \times\left[0, F_{y, \mathcal{P}_{M}^{*}}^{-1}(v)\right]\right),
$$

which is a proper copula distribution.

One good property of such normalization is that it preserves the copula structure due to the monotonicity of the transform, i.e., $\mathcal{P}_{m}^{*}$ and $\tilde{\mathcal{P}}_{m}^{*}$ share the same copula. Further, asymptotically, $F_{x, \mathcal{P}_{m}^{*}}$ and $F_{y, \mathcal{P}_{m}^{*}}$ converge to the uniforms, leading to $\tilde{\mathcal{P}}_{m}^{*} \stackrel{p}{\rightarrow} \mathcal{P}_{m}^{*}$.

\subsection{Estimation with Unknown Marginals}

Throughout this article, especially for the simulations, we focus on the estimation of a copula itself, assuming the marginals are known. Here we address more practical scenarios where the marginals are to be estimated. As we stated earlier, the marginal distributions can be more accurately estimated than the copula as data concentrate to a single dimension. Generally, suppose we have $N$ i.i.d. observations $\left(X_{i}, Y_{i}\right)$, and their marginal distribution estimates are either parametric or nonparametric, denoted by $\hat{F}_{X}$ and $\hat{F}_{Y}$ respectively. The inverse transform $\left(\hat{F}_{X}^{-1}\left(X_{i}\right), \hat{F}_{Y}^{-1}\left(Y_{i}\right)\right)=$ $\left(\hat{U}_{i}, \hat{V}_{i}\right)$ is considered copula-distributed observations where the regular D-P tree copula estimation procedure can be applied. 


\subsection{Higher Dimension}

Most of the results of the D-P tree prior on bivariate copulas can be generalized to higher dimensions. Specifically, for a $d$-dimensional copula, we can generalize the D-P tree prior to $C \sim$ $\operatorname{DPT}(\Pi, \mathcal{A})$, where $\Pi$ is a $2^{d}$-partition on the $d$-dimensional unit cube and the same parametrization for $\mathcal{A}=\left\{\alpha: \alpha_{\epsilon_{1} \ldots \epsilon_{m}}=m^{2}\right\}$. Those properties of a bivariate D-P tree including conjugacy, continuity and convergence, are still preserved.

However, as the dimension increases, the sparsity of data would cause great difficulty for accurate copula estimation, especially among nonparametric settings including the D-P tree prior. Further, though the computational complexity is stable, the D-P tree still requires exponentially increasing storage power as the dimension increases. Yet one potentially favorable feature of the D-P tree that we have observed through simulations is its strong smoothing effect and improved estimation accuracy when the sample size is small. Thereby, the D-P tree prior could be the more favorable nonparametric method compared to other alternatives with sparse observations under higher-dimensional scenarios. This could be one potential angle for further studies.

\section{CONCLUSION}

The proposed Dirichlet-based Pólya tree (D-P tree) prior preserves properties including conjugacy, continuity and convergence as the classic Pólya tree, which provides a foundation for nonparametric copula estimation under the Bayesian framework. Compared with other Bayesian copula estimation methods, the D-P tree prior exhibits strength in robustness and consistency, remedying the severe bias of the earlier Pólya tree-based prior in copula estimation, and also overcoming the inconsistency issue of the family-based mixture model under misspecification. In comparison with the nonparametric methods under the frequentist settings, the D-P tree posterior mean estimator performs competitively well and rather stably across various distance measures. Notably, with a small sample size, the D-P tree copula estimator is advantageous in estimation accuracy, which may imply its potential in higher-dimensional cases where observations are heavily diluted.

However, there still are issues remaining with the D-P tree prior worthy of further exploration, such as the marginal bias caused by the randomness in the prior and a more efficient application in higher dimensions. Further, in terms of the D-P tree's application to the copula prediction of the stock prices, we have not yet fully exploited the timely nature of the data. The exploration of time-dependent D-P tree prior could be of great future research interest. In addition, alternative priors under nonparametric Bayesian frameworks could also be of future interest to overcome the 
limitations of the D-P tree prior.

\section{APPENDIX}

\section{A DEFINITIONS}

\section{A.1 Copula}

Definition 4. $C:[0,1]^{d} \rightarrow[0,1]$ is a d-dimensional copula, if $C$ is a joint cumulative distribution function for a d-dimensional random vector on $[0,1]^{d}$ with uniform marginals. For two-dimensional case, that is, $C(u, v)=P(U \leq u, V \leq v)$, where $U, V \sim U n i f[0,1]$. And the joint density function $c(u, v)$ is called copula density.

Sklar's Theorem (Sklar 1959), if $X$ and $Y$ are random variables with cumulative distribution functions $F$ and $G$, and a joint distribution function $H$, then there exists a copula $C$ such that for all $(x, y) \in \mathbb{R}^{2}, H(x, y)=C(F(x), G(y))$, and for density function, we have $h(x, y)=$ $c(F(x), G(y)) f(x) g(y)$, where $f$ and $g$ are marginal density functions and $h$ is the joint density.

\section{A.2 Pólya Tree}

Definition 5. (Lavine 1992) Let $\Omega$ be a separable measurable space and $\Pi=\left\{B_{\epsilon}\right\}$ be one of its binary tree partitions that generate the measurable sets, where $B_{\emptyset}=\Omega$ and $B_{\epsilon}=B_{\epsilon 0} \cup B_{\epsilon 1}$. A random probability measure $\mathcal{P}$ is said to have a Pólya tree distribution, or Pólya tree prior, with parameters $(\Pi, \mathcal{A})$, written $\mathcal{P} \sim \operatorname{PT}(\Pi, \mathcal{A})$, if there exists non-negative numbers $\mathcal{A}=\left\{\alpha_{0}, \alpha_{1}, \alpha_{00}, \ldots\right\}$ and random variables $\mathcal{Z}=\left\{Z_{0}, Z_{1}, Z_{00} \ldots\right\}$ such that the following hold:

- all the random variables in $\mathcal{Z}$ are independent;

- for every $m=1,2, \ldots$ and every $\epsilon=\epsilon_{1} \epsilon_{2} \ldots \epsilon_{m}, Z_{\epsilon} \sim \operatorname{Beta}\left(\alpha_{\epsilon 0}, \alpha_{\epsilon 1}\right)$;

- for every $\epsilon, \mathcal{P}\left(B_{\epsilon=\epsilon_{1} \epsilon_{2} \ldots \epsilon_{m}}\right)=\left(\prod_{j=1 ; \epsilon_{j}=0}^{m} Z_{\epsilon_{1} \epsilon_{2} \ldots \epsilon_{j-1}}\right)\left\{\prod_{j=1 ; \epsilon_{j}=1}^{m}\left(1-Z_{\epsilon_{1} \epsilon_{2} \ldots \epsilon_{j-1}}\right)\right\}$ where the first terms in the products are interpreted as $Z_{\emptyset} \sim \operatorname{Beta}\left(\alpha_{0}, \alpha_{1}\right)$ and $\left(1-Z_{\emptyset}\right)$.

\section{B PROOFS}

\section{B.1 Proof of Proposition 4}

We consider $\mathcal{P}_{\mathcal{M}}$ on the measurable partition $\left\{B_{\epsilon}\right\}$. For any $B_{k}=B_{\epsilon_{1} \ldots \epsilon_{k}} \in \Pi$, for $M$ large enough, let $B_{j}=B_{\epsilon_{1} \ldots \epsilon_{j}}$, and $B_{1} \subset B_{2} \cdots \subset B_{k}$. 
If $C\left(B_{k}\right)>0$,

$$
\begin{aligned}
\mathrm{E}\left(\mathcal{P}_{M}\left(B_{k}\right) \mid \boldsymbol{Y}\right) & \left.=\prod_{j=1}^{k} \frac{\alpha_{\epsilon_{1} \ldots \epsilon_{j}}+n_{\epsilon_{1} \ldots \epsilon_{j}}}{\sum_{i=0}^{3}\left(\alpha_{\epsilon_{1} \ldots \epsilon_{j-1} i}+n_{\epsilon_{1} \ldots \epsilon_{j-1} i}\right)}=\prod_{j=1}^{k} \frac{\frac{j^{2}}{N}+C\left(B_{j}\right)+O\left(\frac{1}{\sqrt{N}}\right)}{\frac{4 j^{2}}{N}+C\left(B_{j-1}\right)+O\left(\frac{1}{\sqrt{N}}\right)}\right\} \leq C\left(B_{k}\right)+\prod_{j=1}^{k}\left\{1+\frac{3 j^{2}+O(\sqrt{N})}{4 j^{2}+n_{j-1}}\right\}-1 \\
& =\prod_{j=1}^{k}\left\{\frac{C\left(B_{j}\right)}{C\left(B_{j-1}\right)}+\frac{j^{2}-4 j^{2} \frac{C\left(B_{j}\right)}{C\left(B_{j-1}\right)}+O(\sqrt{N})}{4 n_{j-1}}\right\} \\
& =C\left(B_{k}\right)+\exp \left\{\sum_{j=1}^{k} \frac{3 j^{2}+O(\sqrt{N})}{4 j^{2}+n_{j-1}}+O\left(\sum_{j=1}^{k}\left(\frac{3 j^{2}+O(\sqrt{N})}{4 j^{2}+n_{j-1}}\right)^{2}\right)\right\}-1 \\
& =C\left(B_{k}\right)+O\left(\sum_{j=1}^{k} \frac{3 j^{2}+O(\sqrt{N})}{4 j^{2}+n_{j-1}}\right)=C\left(B_{k}\right)+O\left(\sum_{j=1}^{k} \frac{3 j^{2}+O(\sqrt{N})}{4 j^{2}+N C\left(B_{j-1}\right)+O(\sqrt{N})}\right) \\
& \leq C\left(B_{k}\right)+O\left(\sum_{j=1}^{k} \frac{3 j^{2}+O(\sqrt{N})}{N C\left(B_{j-1}\right)}\right)=C\left(B_{k}\right)+\max \left\{O\left(\frac{M}{\sqrt{N}}\right), O\left(\frac{M^{3}}{N}\right)\right\} .
\end{aligned}
$$

If $C\left(B_{k}\right)=0$, suppose $l=\max _{i<k}\left\{C\left(B_{\epsilon_{1} \ldots \epsilon_{i}}\right)>0\right\}$,

$$
\begin{aligned}
\mathrm{E}\left(\mathcal{P}_{M}\left(B_{k}\right) \mid \boldsymbol{Y}\right) & =\prod_{j=1}^{k} \frac{\alpha_{\epsilon_{1} \ldots \epsilon_{j}}+n_{\epsilon_{1} \ldots \epsilon_{j}}}{\sum_{i=0}^{3}\left(\alpha_{\epsilon_{1} \ldots \epsilon_{j-1} i}+n_{\epsilon_{1} \ldots \epsilon_{j-1} i}\right)}=\prod_{j=1}^{l+1} \frac{\frac{j^{2}}{N}+C\left(B_{j}\right)+O\left(\frac{1}{\sqrt{N}}\right)}{\frac{4 j^{2}}{N}+C\left(B_{j-1}\right)+O\left(\frac{1}{\sqrt{N}}\right)}\left(\frac{1}{4}\right)^{M-l-1} \\
\leq & C\left(B_{l+1}\right)\left(\frac{1}{4}\right)^{M-l-1}+\max \left\{O\left(\frac{M}{\sqrt{N}}\right), O\left(\frac{M^{3}}{N}\right)\right\}\left(\frac{1}{4}\right)^{M-l-1}=0+\max \left\{O\left(\frac{M}{\sqrt{N}}\right), O\left(\frac{M^{3}}{N}\right)\right\} \\
\operatorname{var}\left(\mathcal{P}_{M}\left(B_{k}\right) \mid \boldsymbol{Y}\right) & =\operatorname{var}\left(\prod_{j=1}^{k} Z_{\epsilon_{i} \ldots \epsilon_{j}} \mid Y\right)=\operatorname{var}\left(\prod_{j=1}^{k} Z_{j} \mid Y\right)=\mathrm{E}\left(\operatorname{var}\left(Z_{1} \mid Y\right) \prod_{j=2}^{k} Z_{j}^{2} \mid Y\right)+\operatorname{var}\left(\mathrm{E}\left(Z_{1} \mid Y\right) \prod_{j=2}^{k} Z_{j} \mid Y\right) \\
& =\operatorname{var}\left(Z_{1} \mid Y\right) \prod_{j=2}^{k} \operatorname{var}\left(Z_{j}^{2} \mid Y\right)+E^{2}\left(Z_{1} \mid Y\right) \operatorname{var}\left(\prod_{j=2}^{k} Z_{j} \mid Y\right) \\
& \leq \operatorname{var}\left(Z_{1} \mid Y\right)+\operatorname{var}\left(\prod_{j=2}^{k} Z_{j} \mid Y\right) \leq \sum_{j=1}^{k} \operatorname{var}\left(Z_{j} \mid Y\right) \\
& =\sum_{j=1}^{M} \frac{\left(\alpha_{\epsilon_{1} \ldots \epsilon_{j}}+n_{\epsilon_{1} \ldots \epsilon_{j}}\right)\left\{\sum_{i \neq j}\left(\alpha_{\epsilon_{1} \ldots \epsilon_{j-1} i}+n_{\epsilon_{1} \ldots \epsilon_{j-1} i}\right)\right\}}{\left\{\sum_{i=0}^{3}\left(\alpha_{\epsilon_{1} \ldots \epsilon_{j-1} i}+n_{\epsilon_{1} \ldots \epsilon_{j-1} i}\right)\right\}^{2}\left\{\sum_{i=0}^{3}\left(\alpha_{\epsilon_{1} \ldots \epsilon_{j-1} i}+n_{\epsilon_{1} \ldots \epsilon_{j-1} i}\right)+1\right\}} \\
& \leq \sum_{j=1}^{M} \frac{1}{\left\{4 j^{2}+n_{j-1}+1\right\}} \leq \frac{M}{N C\left(B_{k}\right)}=O\left(\frac{M}{N}\right)
\end{aligned}
$$

Thereby for any measurable set $B \subset I$,

$$
\begin{aligned}
\mathrm{E}\left(\mathcal{P}_{M}(B) \mid Y_{N}\right) & \rightarrow C(B), \quad \operatorname{var}\left(\mathcal{P}_{M}(B) \mid Y_{N}\right) \rightarrow 0 \\
P\left(\left|\mathcal{P}_{M \mid Y}(B)-C(B)\right| \geq \epsilon\right) & \leq \frac{E^{2}\left(\mathcal{P}_{M \mid Y}(B)-C(B)\right)+\operatorname{var}\left(\mathcal{P}_{M}(B) \mid Y\right)}{\epsilon^{2}} \rightarrow 0
\end{aligned}
$$




\section{B.2 Proof of Proposition 5}

For any $B_{k}=B_{\epsilon_{1} \ldots \epsilon_{k}}, k \geq M, \mathrm{E}\left(\mathcal{P}_{M}\left(B_{k}\right) \mid \boldsymbol{Y}\right)=\prod_{j=1}^{M} \frac{\alpha_{\epsilon_{1} \ldots \epsilon_{j}}+n_{\epsilon_{1} \ldots \epsilon_{j}}}{\sum_{i=0}^{3}\left(\alpha_{\epsilon_{1} \ldots \epsilon_{j-1}}+n_{\epsilon_{1} \ldots \epsilon_{j-1}}\right)} \prod_{j=M+1}^{k} \frac{1}{4}$. If $C\left(B_{\epsilon_{1} \ldots \epsilon_{k}}\right)>0$ :

$$
\begin{aligned}
\mathrm{E}\left(\mathcal{P}_{M}\left(B_{k}\right) \mid \boldsymbol{Y}\right) & =\left(\frac{1}{4}\right)^{k-M} \prod_{j=1}^{M} \frac{j^{2}+n_{j}}{4 j^{2}+n_{j-1}} \leq\left(\frac{1}{4}\right)^{k-M}\left(C\left(B_{M}\right)+O\left(\sum_{j=1}^{k} \frac{3 j^{2}+O(\sqrt{N})}{N C\left(B_{j-1}\right)}\right)\right) \\
& \leq\left(\frac{1}{4}\right)^{k-M}\left(C\left(B_{M}\right)+\max \left\{O\left(\frac{M}{\sqrt{N} \gamma(M)}\right), O\left(\frac{M^{3}}{N \gamma(M)}\right)\right\}\right) .
\end{aligned}
$$

For $C \in C^{1}([0,1] \times[0,1])$ :

$$
\begin{aligned}
\sup \left|\mathrm{E}\left(\mathcal{P}_{M}\left(B_{k}\right) \mid \boldsymbol{Y}\right)-C\left(B_{k}\right)\right| & \leq\left(\frac{1}{4}\right)^{k} \sup \left|c\left(b_{\epsilon_{1} \ldots \epsilon_{k}}\right)-c\left(b_{\epsilon_{1} \ldots \epsilon_{M}}\right)\right|+\max \left\{O\left(\frac{M}{\sqrt{N} \gamma(M)}\right), O\left(\frac{M^{3}}{N \gamma(M)}\right)\right\} \\
& \leq\left(\frac{1}{4}\right)^{k}(1 / 2)^{k} \sup \left|c^{\prime}\right|+\max \left\{O\left(\frac{M}{\sqrt{N} \gamma(M)}\right), O\left(\frac{M^{3}}{N \gamma(M)}\right)\right\} \\
& =\max \left\{O\left(\frac{M}{\sqrt{N} \gamma(M)}\right), O\left(\frac{M^{3}}{N \gamma(M)}\right)\right\} .
\end{aligned}
$$

If $C\left(B_{k}\right)=0$, suppose $l=\max _{i<k}\left\{C\left(B_{\epsilon_{1} \ldots \epsilon_{i}}\right)>0\right\}$ :

$$
\begin{aligned}
\sup \mathrm{E}\left(\mathcal{P}_{M}\left(B_{k}\right) \mid \boldsymbol{Y}\right) & =\sup \left(\frac{1}{4}\right)^{k-l-1} \prod_{j=1}^{l+1} \frac{\frac{j^{2}}{N}+C\left(B_{j}\right)+O\left(\frac{1}{\sqrt{N}}\right)}{\frac{4 j^{2}}{N}+C\left(B_{j-1}\right)+O\left(\frac{1}{\sqrt{N}}\right)} \leq \sup \left(\frac{1}{4}\right)^{k-l-1}\left(C\left(B_{l+1}\right)+O\left(\sum_{j=1}^{k} \frac{3 j^{2}+O(\sqrt{N})}{N C\left(B_{j-1}\right)}\right)\right) \\
& =0+\max \left\{O\left(\frac{M}{\sqrt{N} \gamma(M)}\right), O\left(\frac{M^{3}}{N \gamma(M)}\right)\right\}
\end{aligned}
$$

Thereby $\sup _{B}\left|\mathrm{E}\left(\mathcal{P}_{M} \mid \boldsymbol{Y}\right)-C\right| \rightarrow 0$.

By the proof of Proposition 4

$$
\sup \operatorname{var}\left(\mathcal{P}_{M}\left(B_{\epsilon}\right) \mid \boldsymbol{Y}\right) \leq \sup O\left(\frac{M}{N C\left(B_{k}\right)}\right) \leq O\left(\frac{M}{N \gamma(M)}\right) .
$$

Let $S_{M}^{\delta}=\left\{B_{\epsilon_{1} \ldots \epsilon_{M}}: \exists x \in B_{\epsilon_{1} \ldots \epsilon_{M}}, c(x)<\delta\right\}, J_{M}^{\delta}=\cup_{B \in S_{M}^{\delta}} B$, thereby $\inf _{I / J_{M}^{\delta}} c(x) \geq \delta$. By $C \in C^{1}(I), \forall \epsilon>0$, for $M$ large enough, $\forall B \in\left\{B_{\epsilon_{1} \ldots \epsilon_{M}}\right\}, x, y \in B,|c(x)-c(y)| \leq \epsilon / 8$, taking $\delta=\epsilon / 4, \epsilon / 4>\sup _{J_{M}^{\epsilon / 8}} c(x)$. Therefore,

$d_{T V}\left(\mathcal{P}_{M \mid Y}, C\right)=\int_{I}\left|p_{M \mid Y}(x)-c(x)\right| d x=\int_{I / J_{M}^{\epsilon / 8}}\left|p_{M \mid Y}(x)-c(x)\right| d x+\int_{J_{M}^{\epsilon / 8}}\left|p_{M \mid Y}(x)-c(x)\right| d x=I_{1}+I_{2}$

where $p_{M \mid Y}$ is the density function of $\mathcal{P}_{M \mid Y}$.

$$
\begin{aligned}
& I_{2}=\int_{J_{M}^{\epsilon / 8}}\left|p_{M \mid Y}(x)-c(x)\right| d x \leq \int_{J_{M}^{\epsilon / 8}} p_{M \mid Y}(x) d x+\int_{J_{M}^{\epsilon / 8}} c(x) d x \leq \int_{I / J_{M}^{\epsilon / 8}}\left|p_{M \mid Y}(x)-c(x)\right| d x+2 \int_{J_{M}^{\epsilon / 8}} c(x) d x . \\
& d_{T V}\left(\mathcal{P}_{M \mid Y}, C\right) \leq 2 \int_{I / J_{M}^{\epsilon / 8}}\left|p_{M \mid Y}(x)-c(x)\right| d x+2 \int_{J_{M}^{\epsilon / 8}} c(x) d x \leq 2 \int_{I / J_{M}^{\epsilon / 8}}\left|p_{M \mid Y}(x)-c(x)\right| d x+\dot{\epsilon} / 2 .
\end{aligned}
$$




$$
\begin{aligned}
I_{1} & =\int_{I / J_{M}^{\epsilon / 8}}\left|p_{M \mid Y}(x)-c(x)\right| d x=\int_{I / J_{M}^{\epsilon / 8}}\left|2^{2 M} P_{M \mid Y}\left(B_{x}\right)-c(x)+c\left(b_{x}\right)-c\left(b_{x}\right)\right| d x \\
& \leq \sum_{B \in\left\{B_{\epsilon_{1} \ldots \epsilon_{M}}\right\} / S_{M}^{\epsilon / 8}}\left\{\left|\mathcal{P}_{M \mid Y}(B)-C(B)\right|+\int_{B}|c(b)-c(x)| d x\right\}
\end{aligned}
$$

where $B_{x} \in\left\{B_{\epsilon_{1} \ldots \epsilon_{M}}\right\}$ such that $x \in B_{x}$, and $C\left(B_{x}\right)=c\left(b_{x}\right) \mu\left(B_{x}\right) . \forall B \in\left\{B_{\epsilon_{1} \ldots \epsilon_{M}}\right\}, b, x \in B$, $|c(b)-c(x)| \leq \epsilon / 8$, we have $\sum_{\left\{B_{\epsilon_{1} \ldots \epsilon_{M}}\right\} / S_{M}^{\epsilon / 8}}\left\{\int_{B}|c(b)-c(x)| d x\right\} \leq \epsilon / 8$.

$$
\begin{aligned}
& P\left(\sum_{\left\{B_{\left.\epsilon_{1} \ldots \epsilon_{M}\right\} / S_{M}^{\epsilon / 8}}\right.}\left|\mathcal{P}_{M \mid Y}(B)-C(B)\right|>\epsilon / 4\right) \leq P\left(\max _{B \in\left\{B_{\epsilon_{1} \ldots \epsilon_{M}}\right\} / S_{M}^{\epsilon / 8}}\left|\mathcal{P}_{M \mid Y}(B)-C(B)\right| \geq \frac{\epsilon}{2^{2 M+2}}\right) \\
& \leq \sum_{B \in\left\{B_{\epsilon_{1} \ldots \epsilon_{M}}\right\} / S_{M}^{\epsilon / 8}} P\left(\left|\mathcal{P}_{M \mid Y}(B)-C(B)\right| \geq \frac{\epsilon}{2^{2 M+2}}\right) \\
& \leq 2^{2 M+2}\left(\frac{2^{2 M}}{\epsilon}\right)^{2}\left\{\sup \left|\mathrm{E}\left(\mathcal{P}_{M \mid Y}(B)\right)-C(B)\right|^{2}+\sup \operatorname{var}\left(\mathcal{P}_{M \mid Y}(B)\right)\right\} \\
& =2^{6 M} \max \left\{O\left(\frac{M}{\sqrt{N} \gamma(M)}\right)^{2}, O\left(\frac{M^{3}}{N \gamma(M)}\right)^{2}, O\left(\frac{M}{N \gamma(M)}\right)\right\} .
\end{aligned}
$$

Note that here $r(M) \sim \min _{\left\{B_{\epsilon_{1} \ldots \epsilon}\right\} / S_{M}^{\epsilon / 8}} C\left(B_{M}\right) \geq \epsilon / 2^{2 M+2}$. Thus, by taking $N \propto O\left(2^{10 M} M^{2+\eta}\right)$, $P\left(d_{T V}\left(\mathcal{P}_{M}, C\right) \geq \epsilon \mid Y\right)=O\left(\frac{1}{M^{\eta}}\right) \rightarrow 0$.

\section{B.3 Proof of Proposition 6}

1. For $c \geq \xi>0, \forall B_{M}, \exists b_{M} \in I$, such that $C\left(B_{M}\right)=2^{-2 M} c\left(b_{M}\right) \geq 2^{-2 M} \xi$, thereby $\gamma \sim 2^{-2 M}$,

2. We assume $\rho<0$, let $\alpha=\Phi^{-1}\left(2^{-M}\right)$, by symmetry of Gaussian copula, for fixed $\rho$,

$$
\begin{aligned}
\gamma(M) & =\int_{-\infty}^{\alpha} \int_{-\infty}^{\alpha} \frac{1}{2 \pi \sqrt{1-\rho^{2}}} \exp \left\{-\frac{x^{2}+y^{2}-2 \rho x y}{2\left(1-\rho^{2}\right)}\right\} d x d y \\
& \geq \int_{-\infty}^{\alpha} \int_{-\infty}^{\alpha} \frac{1}{2 \pi \sqrt{1-\rho^{2}}} \exp \left\{-\frac{(1-\rho)\left(x^{2}+y^{2}\right)}{2\left(1-\rho^{2}\right)}\right\} d x d y=\Phi^{2}(\alpha \sqrt{1+\rho}) \sqrt{\frac{1+\rho}{1-\rho}} \approx 2^{-2 M} .
\end{aligned}
$$

\section{REFERENCES}

Azzalini, A. and Capitanio, A. (1999), "Statistical Applications of the Multivariate Skew Normal Distribution," Journal of the Royal Statistical Society: Series B (Statistical Methodology), 61, $579-602$.

Behnen, K., Hušková, M., and Neuhaus, G. (1985), "Rank Estimators of Scores for Testing Independence," Statistics \& Risk Modeling, 3, 239-262. 
Chen, S. X. and Huang, T.-M. (2007), "Nonparametric Estimation of Copula Functions for Dependence Modelling," Canadian Journal of Statistics, 35, 265-282.

Deheuvels, P. (1979), "La Fonction de Dépendance Empirique et Ses Propriétés. Un Test Non Paramétrique d'Indépendance," Académie Royale de. Belgique. Bulletin de la Classe des Sciences. 6e Série., 65, 274-292.

Devroye, L. and Györfi, L. (1985), Nonparametric Density Estimation: the L1 View, vol. 119 of Wiley Series in Probability and Statistics, New York, NY: Wiley.

Dortet-Bernadet, J.-L. (2005), "Bayesian Inference on Copulas and Tests of Independence," Unpublished manuscript.

Ferguson, T. S. (1974), "Prior Distributions on Spaces of Probability Measures," The Annals of Statistics, 2, 615-629.

Filippi, S. and Holmes, C. C. (in press), "A Bayesian Nonparametric Approach to Testing for Dependence Between Random Variables," Bayesian Analysis.

Gasser, T. and Müller, H.-G. (1979), Smoothing Techniques for Curve Estimation, Heidelberg, Germany: Springer, chap. Kernel Estimation of Regression Functions, pp. 23-68.

Genest, C., Ghoudi, K., and Rivest, L.-P. (1995), "A Semiparametric Estimation Procedure of Dependence Parameters in Multivariate Families of Distributions," Biometrika, 82, 543-552.

Gijbels, I. and Mielniczuk, J. (1990), "Estimating the Density of a Copula Function," Communications in Statistics-Theory and Methods, 19, 445-464.

Hanson, T. E. (2006), "Inference for Mixtures of Finite Pólya Tree Models," Journal of the American Statistical Association, 101, 1548-1565.

Hominal, P. and Deheuvels, P. (1979), "Estimation Non Paramétrique de la Densité Compte-tenu d'Informations sur le Support," Revue de Statistique Appliquée, 27, 47-68.

Jaworski, P., Durante, F., Hardle, W. K., and Rychlik, T. (2010), Copula Theory and Its Applications, Heidelberg, Germany: Springer.

Joe, H. (1997), Multivariate Models and Multivariate Dependence Concepts, Boca Raton, FL: CRC Press. 
John, R. (1984), "Boundary Modification for Kernel Regression," Communications in StatisticsTheory and Methods, 13, 893-900.

Lavine, M. (1992), "Some Aspects of Pólya Tree Distributions for Statistical Modelling," The Annals of Statistics, 20, 1222-1235.

Müller, H.-G. (1991), "Smooth Optimum Kernel Estimators Near Endpoints," Biometrika, 78, $521-530$.

Nelsen, R. B. (2007), An Introduction to Copulas, New York, NY: Springer.

Oakes, D. (1982), "A Model for Association in Bivariate Survival Data," Journal of the Royal Statistical Society. Series B (Methodological), 44, 414-422.

- (1986), "Semiparametric Inference in a Model for Association in Bivanate Survival Data," Biometrika, 73, 353-361.

Paddock, S. M., Ruggeri, F., Lavine, M., and West, M. (2003), "Randomized Polya tree models for nonparametric Bayesian inference," Statistica Sinica, 13, 443-460.

Scaillet, O., Charpentier, A., and Fermanian, J.-D. (2007), "The Estimation of Copulas: Theory and Practice," Copulas: from Theory to Applications in Finance, 35-62.

Schervish, M. J. (1995), Theory of Statistics, New York, NY: Springer.

Schuster, E. F. (1985), "Incorporating Support Constraints into Nonparametric Estimators of Densities," Communications in Statistics-Theory and Methods, 14, 1123-1136.

Schweizer, B. and Wolff, E. F. (1981), "On Nonparametric Measures of Dependence for Random Variables," The Annals of Statistics, 9, 879-885.

Sklar, A. (1959), Fonctions de Répartition à n Dimensions et Leurs Marges, Paris, France: Université Paris 8.

Wong, W. H., Ma, L., et al. (2010), "Optional Pólya Tree and Bayesian Inference," The Annals of Statistics, 38, 1433-1459.

Wu, J., Wang, X., and Walker, S. G. (2014), "Bayesian Nonparametric Inference for a Multivariate Copula Function," Methodology and Computing in Applied Probability, 16, 747-763. 


\section{SUPPLEMENTARY MATERIAL}

\section{S.1 COMMON COPULAS}

\section{S.1.1 Gaussian Copula}

The copula density of a bivariate Gaussian copula is given by

$$
c(u, v)=\frac{1}{\sqrt{1-\rho^{2}}} \exp \left\{-\frac{\rho^{2}\left(x^{2}+y^{2}\right)-2 \rho x y}{2\left(1-\rho^{2}\right)}\right\},
$$

where $\rho \in[-1,1]$ is the correlation parameter of the copula, $x=\Phi^{-1}(u), y=\Phi^{-1}(v)$, and $\Phi^{-1}$ is the inverse of the standard univariate Gaussian CDF.

\section{S.1.2 Student's t Copula}

The copula density of a bivariate Student's t-copula follows

$$
c(u, v)=\frac{\Gamma\left(\frac{\nu+2}{2}\right) / \Gamma\left(\frac{\nu}{2}\right)}{\nu \pi f_{t_{\nu}}(x) f_{t_{\nu}}(y) \sqrt{1-\rho^{2}}}\left\{1+\frac{x^{2}+y^{2}-2 \rho x y}{\nu\left(1-\rho^{2}\right)}\right\}^{-\frac{\nu+1}{2}},
$$

where the two parameters, the correlation $\rho \in[-1,1]$ and the degree of freedom $\nu>0, x=$ $F_{t_{\nu}}(u), y=F_{t_{\nu}}(v)$, and $f_{t_{\nu}}$ and $F_{t_{\nu}}$ are the PDF and CDF of the standard univariate Student's t-distribution with the degree of freedom of $\nu$.

\section{S.1.3 Gumbel Copula}

The copula density of a bivariate Gumbel copula is given by

$$
\begin{gathered}
c(u, v)=C(u, v)(u v)^{-1}\left[\{-\log (u)\}^{a}+\{-\log (v)\}^{a}\right]^{-2+2 / a}\{\log (u) \log (v)\}^{a-1} \\
\left(1+(a-1)\left[\{-\log (u)\}^{a}+\{-\log (v)\}^{a}\right]^{-1 / a}\right),
\end{gathered}
$$

where $a \geq 1$ is the dependence parameter.

\section{S.1.4 Skew-normal Copula}

A d-dimensional random vector $\boldsymbol{Z}=\left(Z_{1}, \ldots, Z_{d}\right)^{T}$ follows a skew-normal distribution Azzalini and Capitanio 1999), denoted $\boldsymbol{Z} \sim S N_{d}(\Omega, \boldsymbol{\alpha})$ if

$$
\boldsymbol{Z}= \begin{cases}\boldsymbol{X} & \text { if } X_{0}>0 \\ -\boldsymbol{X} & \text { otherwise }\end{cases}
$$


where $\left(X_{0}, \boldsymbol{X}\right)^{T} \sim N_{d+1}\left(\mathbf{0}, \Omega^{*}\right), \Omega^{*}=\left(\begin{array}{cc}1 & \boldsymbol{\delta}^{T} \\ \boldsymbol{\delta} & \Omega\end{array}\right)$ and $\boldsymbol{\alpha}=\frac{1}{\left(1-\boldsymbol{\delta}^{T} \Omega^{-1} \boldsymbol{\delta}\right)^{1 / 2}} \Omega^{-1} \boldsymbol{\delta}$. And the density functions for $\boldsymbol{Z}$ is $f_{S N, d}(\boldsymbol{z} ; \Omega, \boldsymbol{\alpha})=2 \phi_{d}(\boldsymbol{z} ; \Omega) \Phi\left(\boldsymbol{\alpha}^{T} \boldsymbol{z}\right)$, where $\phi_{d}(\cdot ; \Omega)$ is the $d$-dimensional normal density with zero mean and correlation matrix $\Omega$.

For the marginals, suppose $\boldsymbol{Z}$ is partitioned as $\boldsymbol{Z}=\left(\boldsymbol{Z}_{1}^{T}, \boldsymbol{Z}_{2}^{T}\right)^{T}$ of dimension $h$ and $d-h ; \Omega$ and $\boldsymbol{\alpha}$ by

$$
\Omega=\left(\begin{array}{ll}
\Omega_{11} & \Omega_{12} \\
\Omega_{21} & \Omega_{22}
\end{array}\right), \boldsymbol{\alpha}=\left(\begin{array}{l}
\boldsymbol{\alpha}_{1} \\
\boldsymbol{\alpha}_{2}
\end{array}\right),
$$

then the marginal distribution of $\boldsymbol{Z}_{1}$ is $S N_{d}\left(\Omega_{11}, \overline{\boldsymbol{\alpha}}_{1}\right)$, where

$$
\overline{\boldsymbol{\alpha}}_{1}=\frac{\boldsymbol{\alpha}_{1}+\Omega_{11}^{-1} \Omega_{12} \boldsymbol{\alpha}_{2}}{\left(1+\boldsymbol{\alpha}^{T} \Omega_{22 \cdot 1} \boldsymbol{\alpha}_{2}\right)^{1 / 2}}, \Omega_{22 \cdot 1}=\Omega_{22}-\Omega_{21} \Omega_{21}^{-1} \Omega_{12} .
$$

So the bivariate skew-normal copula density is

$$
c(u, v)=\frac{\phi_{2}\left((x, y)^{T}, \Omega\right) \Phi\left(\alpha_{1} x+\alpha_{2} y\right)}{2 \phi(x) \Phi\left(\bar{\alpha}_{1} x\right) \phi(y) \Phi\left(\bar{\alpha}_{2} y\right)},
$$

where $\Omega=\left(\begin{array}{ll}1 & \rho \\ \rho & 1\end{array}\right) ; \rho \in[-1,1], \alpha_{1}, \alpha_{2}$ are parameters, $x=F_{S N_{1}, \bar{\alpha}_{1}}^{-1}(u), y=F_{S N_{1}, \bar{\alpha}_{2}}^{-1}(v)$, and $F_{S N_{1}, \alpha}$ is the CDF of $Z \sim S N_{1}(1, \alpha)$.

\section{S.2 ADDITIONAL SIMULATION RESULTS}

\begin{tabular}{rrr|ll|ll|ll}
\hline \hline & & & \multicolumn{6}{|c}{$\mathrm{N}$} \\
\cline { 4 - 9 } & $\rho$ & $\alpha$ & \multicolumn{2}{|c|}{1000} & \multicolumn{2}{c}{10,000} & \multicolumn{1}{c}{100,000} \\
\hline 1 & 0.50 & $(2,0)$ & 0.12 & 0.00 & 0.07 & 0.00 & 0.04 & $\mathrm{xx}$ \\
2 & 0.90 & $(2,0)$ & 0.18 & 0.01 & 0.09 & 0.01 & 0.04 & $\mathrm{xx}$ \\
3 & 0.50 & $(-10,50)$ & 0.18 & 0.07 & 0.09 & 0.06 & 0.05 & $\mathrm{xx}$ \\
4 & 0.90 & $(-10,50)$ & 0.21 & 0.03 & 0.10 & 0.03 & 0.05 & $\mathrm{xx}$ \\
5 & 0.50 & $(50,0)$ & 0.12 & 0.01 & 0.07 & 0.00 & 0.04 & $\mathrm{xx}$ \\
6 & 0.90 & $(50,0)$ & 0.16 & 0.05 & 0.08 & 0.05 & 0.04 & $\mathrm{xx}$ \\
7 & 0.50 & $(100,-100)$ & 0.26 & 0.17 & 0.14 & 0.17 & 0.07 & 0.17 \\
8 & 0.90 & $(100,-100)$ & 0.46 & 0.16 & 0.20 & 0.16 & 0.08 & 0.16 \\
\hline \hline
\end{tabular}

Table S.1: Comparison of the K-L divergence between the D-P tree (left) and the Gaussian mixture (right) estimation for skew-normal target copulas. 


\begin{tabular}{|c|c|c|c|c|c|c|c|c|}
\hline$N$ & D-P Tree & Empirical & Kernel & Hist, & D-P Tree & Empirical & Kernel & Hist. \\
\hline & \multicolumn{4}{|c|}{$\rho=0.5, \alpha=(-10,50)$} & \multicolumn{4}{|c|}{$\rho=0.5, \alpha=(100,-100)$} \\
\hline 10 & 0.242 & NA & 0.337 & Inf & 0.528 & $\mathrm{NA}$ & 0.528 & Inf \\
\hline 20 & 0.223 & NA & 0.247 & Inf & 0.473 & NA & 0.428 & Inf \\
\hline 50 & 0.178 & NA & 0.165 & $\operatorname{Inf}$ & 0.386 & NA & 0.314 & Inf \\
\hline 100 & 0.161 & NA & 0.123 & Inf & 0.349 & NA & 0.261 & Inf \\
\hline 500 & 0.098 & NA & 0.064 & $\operatorname{Inf}$ & 0.222 & NA & 0.166 & Inf \\
\hline 1,000 & 0.085 & NA & 0.047 & Inf & 0.184 & NA & 0.136 & Inf \\
\hline 5,000 & 0.056 & NA & 0.027 & $\operatorname{Inf}$ & 0.112 & NA & 0.090 & Inf \\
\hline \multirow[t]{2}{*}{10,000} & 0.041 & NA & 0.020 & $\operatorname{Inf}$ & 0.089 & NA & 0.076 & $\operatorname{Inf}$ \\
\hline & \multicolumn{4}{|c|}{$\rho=0.9, \alpha=(-10,50)$} & \multicolumn{4}{|c|}{ 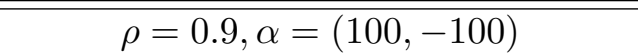 } \\
\hline 10 & 0.441 & NA & 0.449 & Inf & 1.068 & NA & 1.099 & Inf \\
\hline 20 & 0.38 & NA & 0.317 & Inf & 1.013 & NA & 0.969 & Inf \\
\hline 50 & 0.328 & NA & 0.225 & $\operatorname{Inf}$ & 0.836 & NA & 0.763 & Inf \\
\hline 100 & 0.28 & NA & 0.146 & Inf & 0.715 & NA & 0.619 & Inf \\
\hline 500 & 0.163 & NA & 0.065 & $\operatorname{Inf}$ & 0.479 & NA & 0.410 & Inf \\
\hline 1,000 & 0.121 & NA & 0.044 & Inf & 0.379 & NA & 0.345 & Inf \\
\hline 5,000 & 0.068 & NA & 0.021 & $\operatorname{Inf}$ & 0.209 & NA & 0.227 & Inf \\
\hline 10,000 & 0.051 & NA & 0.013 & Inf & 0.164 & NA & 0.191 & Inf \\
\hline
\end{tabular}

Table S.2: Comparison of the K-L divergence between the D-P tree posterior mean estimator and the frequentist estimators for skew-normal target copulas.

\begin{tabular}{|c|c|c|c|c|c|c|c|c|}
\hline$N$ & D-P Tree & Empirical & Kernel & Hist. & D-P Tree & Empirical & Kernel & Hist. \\
\hline & \multicolumn{4}{|c|}{$\rho=0.5, \alpha=(-10,50)$} & \multicolumn{4}{|c|}{$\rho=0.5, \alpha=(100,-100)$} \\
\hline 10 & 0.773 & NA & 2.742 & 93.588 & 1.365 & NA & 2.190 & 71.788 \\
\hline 20 & 0.814 & NA & 1.926 & 55.190 & 1.163 & NA & 2.657 & 56.726 \\
\hline 50 & 0.728 & NA & 1.690 & 35.150 & 1.050 & NA & 1.177 & 36.757 \\
\hline 100 & 0.918 & NA & 1.350 & 25.870 & 1.159 & NA & 1.347 & 25.723 \\
\hline 500 & 0.604 & NA & 0.526 & 11.437 & 1.072 & NA & 1.398 & 11.665 \\
\hline 1,000 & 0.528 & NA & 0.487 & 8.133 & 0.894 & NA & 0.703 & 8.078 \\
\hline 5,000 & 0.525 & NA & 0.412 & 3.617 & 0.703 & NA & 0.516 & 3.601 \\
\hline \multirow[t]{2}{*}{10,000} & 0.389 & NA & 0.263 & 2.565 & 0.701 & NA & 0.769 & 2.600 \\
\hline & \multicolumn{4}{|c|}{$\rho=0.9, \alpha=(-10,50)$} & \multicolumn{4}{|c|}{$\rho=0.9, \alpha=(100,-100)$} \\
\hline 10 & 1.195 & $\mathrm{NA}$ & 2.365 & 74.140 & 2.281 & $\mathrm{NA}$ & 7.372 & 86.920 \\
\hline 20 & 1.297 & NA & 1.837 & 54.820 & 3.335 & NA & 6.047 & 53.928 \\
\hline 50 & 1.521 & NA & 1.689 & 34.064 & 2.217 & NA & 2.185 & 36.227 \\
\hline 100 & 1.640 & NA & 1.427 & 24.826 & 2.098 & NA & 2.380 & 25.320 \\
\hline 500 & 1.132 & NA & 0.951 & 11.515 & 1.980 & NA & 1.826 & 11.277 \\
\hline 1,000 & 0.838 & NA & 0.994 & 8.073 & 1.765 & NA & 1.535 & 8.204 \\
\hline 5,000 & 0.700 & NA & 0.420 & 3.643 & 1.540 & NA & 1.146 & 3.694 \\
\hline 10,000 & 0.597 & NA & 0.300 & 2.575 & 1.949 & NA & 1.177 & 2.843 \\
\hline
\end{tabular}

Table S.3: Comparison of the $\sqrt{M I S E}$ between the D-P tree posterior mean estimator and the frequentist estimators for skew-normal target copulas. 


\begin{tabular}{|c|c|c|c|c|c|c|c|c|}
\hline$N$ & D-P Tree & Empirical & Kernel & Hist. & D-P Tree & Empirical & Kernel & Hist. \\
\hline & \multicolumn{4}{|c|}{$\rho=0.5, \alpha=(-10,50)$} & \multicolumn{4}{|c|}{$\rho=0.5, \alpha=(100,-100)$} \\
\hline 10 & 0.064 & 0.120 & 0.083 & 0.120 & 0.072 & 0.118 & 0.091 & 0.117 \\
\hline 20 & 0.057 & 0.083 & 0.065 & 0.083 & 0.065 & 0.082 & 0.068 & 0.082 \\
\hline 50 & 0.044 & 0.057 & 0.048 & 0.057 & 0.044 & 0.057 & 0.050 & 0.057 \\
\hline 100 & 0.027 & 0.037 & 0.029 & 0.037 & 0.037 & 0.041 & 0.038 & 0.041 \\
\hline 500 & 0.016 & 0.018 & 0.016 & 0.018 & 0.018 & 0.017 & 0.017 & 0.017 \\
\hline 1,000 & 0.013 & 0.013 & 0.013 & 0.013 & 0.013 & 0.012 & 0.013 & 0.012 \\
\hline 5,000 & 0.006 & 0.006 & 0.006 & 0.006 & 0.007 & 0.006 & 0.007 & 0.006 \\
\hline \multirow[t]{2}{*}{10,000} & 0.004 & 0.004 & 0.005 & 0.004 & 0.005 & 0.004 & 0.005 & 0.004 \\
\hline & \multicolumn{4}{|c|}{$\rho=0.9, \alpha=(-10,50)$} & \multicolumn{4}{|c|}{$\bar{\rho} \rho=0.9, \alpha=(100,-100)$} \\
\hline 10 & 0.080 & 0.129 & 0.102 & 0.129 & 0.080 & 0.121 & 0.102 & 0.121 \\
\hline 20 & 0.065 & 0.089 & 0.072 & 0.089 & 0.075 & 0.101 & 0.089 & 0.101 \\
\hline 50 & 0.055 & 0.060 & 0.054 & 0.060 & 0.046 & 0.056 & 0.051 & 0.056 \\
\hline 100 & 0.038 & 0.038 & 0.037 & 0.038 & 0.037 & 0.036 & 0.036 & 0.036 \\
\hline 500 & 0.020 & 0.018 & 0.019 & 0.018 & 0.021 & 0.018 & 0.020 & 0.018 \\
\hline 1,000 & 0.014 & 0.014 & 0.014 & 0.014 & 0.014 & 0.013 & 0.014 & 0.013 \\
\hline 5,000 & 0.006 & 0.006 & 0.006 & 0.006 & 0.007 & 0.005 & 0.008 & 0.005 \\
\hline 10,000 & 0.005 & 0.004 & 0.005 & 0.004 & 0.005 & 0.004 & 0.006 & 0.004 \\
\hline
\end{tabular}

Table S.4: Comparison of the $\sqrt{M I S E_{C}}$ between the D-P tree posterior mean estimator and the frequentist estimators for the skew-normal target copulas.

\begin{tabular}{|c|c|c|c|c|c|c|c|c|}
\hline & \multicolumn{4}{|c|}{$\rho=0.5, \alpha=(-10,50)$} & \multicolumn{4}{|c|}{$\rho=0.5, \alpha=(100,-100)$} \\
\hline$N$ & D-P Tree & Empirical & Kernel & Hist. & D-P Tree & Empirical & Kernel & Hist. \\
\hline 10 & 0.026 & 0.317 & 0.028 & 0.317 & 0.054 & 0.321 & 0.054 & 0.321 \\
\hline 20 & 0.026 & 0.225 & 0.028 & 0.225 & 0.054 & 0.230 & 0.055 & 0.230 \\
\hline 50 & 0.026 & 0.144 & 0.026 & 0.144 & 0.054 & 0.151 & 0.054 & 0.151 \\
\hline 100 & 0.026 & 0.103 & 0.026 & 0.103 & 0.054 & 0.113 & 0.054 & 0.113 \\
\hline 500 & 0.026 & 0.051 & 0.026 & 0.051 & 0.053 & 0.070 & 0.053 & 0.070 \\
\hline 1,000 & 0.026 & 0.041 & 0.026 & 0.041 & 0.053 & 0.062 & 0.053 & 0.062 \\
\hline 5,000 & 0.026 & 0.029 & 0.026 & 0.029 & 0.053 & 0.055 & 0.053 & 0.055 \\
\hline \multirow[t]{2}{*}{10,000} & 0.026 & 0.027 & 0.026 & 0.027 & 0.053 & 0.054 & 0.053 & 0.054 \\
\hline & \multicolumn{4}{|c|}{$\rho=0.9, \alpha=(-10,50)$} & \multicolumn{4}{|c|}{$\rho=0.9, \alpha=(100,-100)$} \\
\hline$N$ & D-P Tree & Empirical & Kernel & Hist. & D-P Tree & Empirical & Kernel & Hist. \\
\hline 10 & 0.010 & 0.316 & 0.015 & 0.316 & 0.063 & 0.322 & 0.065 & 0.322 \\
\hline 20 & 0.010 & 0.224 & 0.014 & 0.224 & 0.063 & 0.232 & 0.064 & 0.232 \\
\hline 50 & 0.010 & 0.142 & 0.012 & 0.142 & 0.063 & 0.155 & 0.063 & 0.155 \\
\hline 100 & 0.010 & 0.100 & 0.011 & 0.100 & 0.063 & 0.118 & 0.063 & 0.118 \\
\hline 500 & 0.010 & 0.046 & 0.009 & 0.046 & 0.063 & 0.077 & 0.063 & 0.077 \\
\hline 1,000 & 0.009 & 0.033 & 0.009 & 0.033 & 0.063 & 0.070 & 0.063 & 0.070 \\
\hline 5,000 & 0.009 & 0.017 & 0.009 & 0.017 & 0.063 & 0.064 & 0.063 & 0.064 \\
\hline 10,000 & 0.009 & 0.013 & 0.009 & 0.013 & 0.063 & 0.063 & 0.063 & 0.063 \\
\hline
\end{tabular}

Table S.5: Comparison of the $\sqrt{M S E_{g}}$ between the D-P tree posterior mean estimator and the frequentist estimators for the skew-normal target copulas. 